Version September 13, 2018

Preprint typeset using $\mathrm{LAT}_{\mathrm{E}} \mathrm{X}$ style emulateapj v. 08/22/09

\title{
STRONG EROSION-DRIVEN NONGRAVITATIONAL EFFECTS IN ORBITAL MOTIONS OF THE KREUTZ SUNGRAZING SYSTEM'S DWARF COMETS
}

\author{
ZdeneK SeKanina ${ }^{1}$ \& Rainer Kracht ${ }^{2}$ \\ ${ }^{1}$ Jet Propulsion Laboratory, California Institute of Technology, 4800 Oak Grove Drive, Pasadena, CA 91109, U.S.A. \\ ${ }^{2}$ Ostlandring 53, D-25335 Elmshorn, Schleswig-Holstein, Germany \\ Version September 13, 2018
}

\begin{abstract}
We investigate the relationships among the angular orbital elements - the longitude of the ascending node $\Omega$, the inclination $i$, and the argument of perihelion, $\omega$ - of the Kreutz system's faint, dwarf sungrazers observed only with the $S O H O / S T E R E O$ coronagraphs; their published orbits were derived using a parabolic, purely gravitational approximation. In a plot of $i$ against $\Omega$ the bright Kreutz sungrazers (such as C/1843 D1, C/1882 R1, C/1963 R1, etc.) fit a curve of fixed apsidal orientation, whereas the dwarf members are distributed along a curve that makes with the apsidal curve an angle of $15^{\circ}$. The dwarf sungrazers' perihelion longitude is statistically invariable, but their perihelion latitude increases systematically with $\Omega$. We find that this trend can be explained by a strong erosiondriven nongravitational acceleration normal to the orbit plane, confirmed for several test dwarf Kreutz sungrazers by orbital solutions with nongravitational terms incorporated directly in the equations of motion on a condition of fixed apsidal orientation. Proceeding in three steps, we first apply Marsden et al.'s standard formalism, solving for the normal acceleration only, and eventually relax additional constraints on the nongravitational law and the acceleration's radial and transverse components. The resulting nongravitational accelerations on the dwarf sungrazers exceed the maximum for catalogued comets in nearly-parabolic orbits by up to three orders of magnitude, topping in exceptional cases the Sun's gravitational acceleration! A mass-loss model suggests that the dwarf sungrazers' nuclei fragment copiously and their dimensions diminish rapidly near the Sun, implying the objects' imminent demise shortly before they reach perihelion.

Subject headings: comets: general - comets: individual (C/1843 D1, C/1880 C1, C/1882 R1, C/1887 B1, C/1945 X1, C/1963 R1, C/1965 S1, C/1970 K1, C/1993 A1, D/1993 F2, C/1998 P1, C/2001 Y4, C/2003 Q7, C/2006 J9, C/2007 X3, C/2007 X13, C/2008 K8, C/2008 M4, C/2008 M5, C/2009 L5, C/2011 W3) — methods: data analysis
\end{abstract}

\section{INTRODUCTION}

The Kreutz system of sungrazers is by far the most prominent ensemble of closely related comets. Named after the German astronomer for his monumental work on the orbital properties of its 19th century and earlier members (Kreutz 1888, 1891, 1901), this comet system is unique. Dynamically, the most peculiar attribute of its members is their extremely close approach to the Sun at perihelion, when the heliocentric distance in an overwhelming number of cases is well below $\sim 2 R_{\odot}\left(1 R_{\odot}=\right.$ 1 solar radius $=0.0046548 \mathrm{AU}$ ) or just about $0.01 \mathrm{AU}$. Due to perturbations, the perihelion distance can become less than the Sun's radius, even though a de facto collision with the Sun is prevented by disintegration.

All Kreutz sungrazers move about the Sun in retrograde orbits, with an inclination in a range of $130^{\circ}-150^{\circ}$. Their orbital periods are, to the extent we can state, based on a few quality data available, probably between $\sim 600$ and $\sim 1000$ years. The angular elements, pinpointing the spatial positions of the sungrazers' orbital planes, may vary from object to object by up to at least $40^{\circ}$ and are subjected to the indirect perturbations by the planets, Jupiter in particular. In contrast, the apsidal line orientation, as derived for the bright members with reliable orbits (also referred to hereafter as the major sungrazers, such as C/1843 D1, C/1882 R1, C/1963 R1, etc.),

Electronic address: Zdenek.Sekanina@jpl.nasa.gov is essentially invariable, with trivial scatter (Sec. 2). It is this fixed position of the line of apsides that Marsden (2005) regarded as a paramount condition for defining the Kreutz system's membership. This definition reflects a common origin of all Kreutz sungrazers and its significance is supported both by Marsden's (1967) study of the indirect planetary perturbations (resulting in a deviation, from one return to perihelion to the next, of typically a few tenths of a degree in the apsidal line) and by Sekanina's (2002) computation of perturbations due to cascading fragmentation of Kreutz comets along the orbit (similarly resulting in a difference of up to $0^{\circ} .02$ per event for a typical separation velocity of $\sim 1 \mathrm{~m} \mathrm{~s}^{-1}$ ).

No Kreutz sungrazer bright enough to observe from the ground appeared between 1970 and 2011. However, 19 fainter members were detected with coronagraphs on board two satellites between 1979 and 1989 (see Marsden 2005 for a review). This activity expanded dramatically following the launch, in late 1995, of the Solar and $\mathrm{He}$ liospheric Observatory (SOHO; Brueckner et al. 1995); over 2000 Kreutz sungrazers have so far been discovered, mostly by amateur astronomers, in images taken with the $\mathrm{C} 2$ and $\mathrm{C} 3$ coronagraphs on board $\mathrm{SOHO}$ since January 1996. None of these faint members of the Kreutz system, which we hereafter refer to as dwarf sungrazers, survived perihelion and none of them achieved a peak brightness greater than apparent magnitude of about -0.5 (e.g., Sekanina \& Kracht 2013 and the references therein). 
The beginnings of the consensus on a common origin of all Kreutz sungrazers date back to the 19th century. This paradigm has been strengthened by more recent developments extensively reviewed by Marsden (2005) in a paper, which the reader is referred to for details. Accordingly, it is fitting to require that the invariable spatial orientation of the apsidal line, typical for the Kreutz system's bright members, be exhibited by all dwarf sungrazers as well. Strangely, this fundamental issue has never been addressed in any detail.

Purely gravitational parabolic orbits for about 1600 dwarf Kreutz comets, observed with the coronagraphs on board $S O H O$ and the Solar Terrestrial Relations Observatory (STEREO; Howard et al. 2008) between early 1996 and mid-2010 were single-handedly computed by Marsden. ${ }^{1}$ This is a homogeneous set of orbits suitable for an in-depth study of apsidal-line orientation, even though the quality of astrometric positions measured from $S O H O$ and $S T E R E O$ images is inferior (because of a large pixel size) compared to the quality of groundbased observations. One also should expect larger uncertainties in the orbital elements of the dwarf sungrazers because of their short orbital arcs under observation, but not any systematic trends.

Even though the orbital elements of nearly 100 additional dwarf Kreutz sungrazers, observed with $\mathrm{SOHO}$ and STEREO in the second half of 2010, have recently been published by Gray (2013), we exclude these from our investigation to avoid mixing different orbit determination approaches.

In this paper we employ the relationship among the angular elements - the longitude of the ascending node, $\Omega$; the inclination, $i$; and the argument of perihelion, $\omega$ - of the 1600 dwarf sungrazers to examine in detail their compliance with the condition of fixed apsidal orientation and to investigate the forces that affect their motions near the Sun. The apsidal orientations of the bright and dwarf Kreutz comets are derived, respectively, in Secs. 2 and 3, while a perturbation analysis of momentum changes in the orbital motions of the dwarf sungrazers is presented in Sec. 4. Sec. 5 compares potential interpretations of detected variations in the apsidal orientation of the dwarf sungrazers, including the role of erosion-driven nongravitational effects, and Sec. 6 displays and explains the distribution of apsidal orientations from gravitational orbital solutions. Further refinements in a model for determining the magnitude of an erosion-driven acceleration are introduced in Secs. 7 and 8, where implications of the findings are also addressed. Sec. 9 offers a summary and conclusions, followed by our thoughts for future work in Sec. 10.

\section{LINE OF REFERENCE APSIDAL ORIENTATION}

High-quality sets of orbital elements are available only for seven bright Kreutz sungrazers seen since the 1840s. All of them were more or less spectacular objects visible with the unaided eye and observed astrometrically over mostly extended periods of time from the ground, and their orbits have the lines of apsides nearly perfectly

\footnotetext{
${ }^{1}$ Most orbits are published in the Catalogue of Cometary Orbits (Marsden \& Williams 2008), with the rest appearing in numerous Minor Planet Circulars in the batches issued between July 2008 and November 2010; see http://www.minorplanetcenter.net/iau/ ECS/MPCArchive/MPCArchive_TBL.html.
}

Table 1

Longitude and Latitude of Perihelion for Kreutz System's Bright Members with Best Determined Orbits

\begin{tabular}{|c|c|c|c|}
\hline $\begin{array}{l}\text { Comet or } \\
\text { fragment }\end{array}$ & $\begin{array}{l}\text { Perihelion } \\
\text { longitude, } \\
L_{\pi}(2000)\end{array}$ & $\begin{array}{c}\text { Perihelion } \\
\text { latitude, } \\
B_{\pi}(2000)\end{array}$ & $\begin{array}{l}\text { Author(s) of } \\
\text { orbital elements }\end{array}$ \\
\hline $\mathrm{C} / 1843 \mathrm{D} 1^{\mathrm{a}}$ & 282.58 & $+35^{\circ} .29$ & $\begin{array}{l}\text { Sekanina \& } \\
\text { Chodas (2008) }\end{array}$ \\
\hline $\mathrm{C} / 1880 \mathrm{C} 1^{\mathrm{b}}$ & 282.38 & +35.25 & Kreutz (1901) \\
\hline C/1882 R1-A ${ }^{\mathrm{c}}$ & 282.94 & +35.23 & Kreutz (1891) \\
\hline $\mathrm{C} / 1882 \mathrm{R} 1-\mathrm{B}^{\mathrm{c}, \mathrm{d}}$ & 282.94 & +35.23 & $"$ \\
\hline C/1882 R1-C & 282.93 & +35.23 & $"$ \\
\hline $\mathrm{C} / 1882 \mathrm{R} 1-\mathrm{D}^{\mathrm{c}}$ & 282.94 & +35.23 & $"$ \\
\hline C/1963 R1 & 282.65 & +35.33 & Marsden (1967) \\
\hline $\mathrm{C}^{\prime} / 1965 \mathrm{~S} 1-\mathrm{A}^{\mathrm{e}}$ & 282.95 & +35.22 & $"$ \\
\hline C/1965 S1-B & 282.96 & +35.22 & $"$ \\
\hline $\mathrm{C} / 1970 \mathrm{~K} 1$ & 282.95 & +35.07 & Marsden (1970) \\
\hline C/2011 W3 & 282.98 & +35.09 & $\begin{array}{l}\text { Sekanina \& } \\
\text { Chodas (2012) }\end{array}$ \\
\hline Average $^{f}$ & $\begin{array}{r}282.81 \\
\pm 0.21\end{array}$ & $\begin{array}{r}+35.22 \\
\pm 0.08\end{array}$ & \\
\hline
\end{tabular}

a Solution II.

b Solution B.

c Solution III.

d Nonrelativistic solution; a relativistic solution by Hufnagel (1919) differs at most in the fifth decimal.

e Nonrelativistic solution; a relativistic solution by Marsden (1967) differs at most in the fourth decimal.

${ }^{f}$ Companions A, C, and D of comet C/1882 R1 and companion B of comet C/1965 S1 are given half weight.

aligned. Table 1, which lists their perihelion longitude $L_{\pi}$ and latitude $B_{\pi}$, shows that a mean perihelion point is described by

$$
\begin{aligned}
& \left\langle L_{\pi}\right\rangle=282^{\circ} .8 \pm 0^{\circ} .2, \\
& \left\langle B_{\pi}\right\rangle=+35^{\circ} .2 \pm 0^{\circ} .1
\end{aligned}
$$

(Equinox J2000.0). The maximum deviations from the adopted mean values amount to $0^{\circ} .4$ in the longitude and less than $0^{\circ} .2$ in the latitude. The curve that in plots of $i$ against $\Omega$ and of $\omega$ against $\Omega$ fits the coordinates (1) is hereafter called the line of reference apsidal orientation and is expressed, respectively in the two plots, by

$$
\begin{aligned}
\cot i & =\cot \left\langle B_{\pi}\right\rangle \sin \left(\left\langle L_{\pi}\right\rangle-\Omega\right), \\
\cos \omega & =\cos \left\langle B_{\pi}\right\rangle \cos \left(\left\langle L_{\pi}\right\rangle-\Omega\right) .
\end{aligned}
$$

Excluded from Table 1 are the headless object C/1887 B1 and $\mathrm{C} / 1945 \mathrm{X} 1$, whose orbit is known less accurately: the solution that Marsden (1967) considered the best differs from the mean by $+0^{\circ} .7$ in $L_{\pi}$ and $+0^{\circ} .8$ in $B_{\pi}$.

\section{RELATIONSHIPS AMONG THE ANGULAR ELEMENTS OF THE DWARF KREUTZ SUNGRAZERS}

A near-perfect alignment of the lines of apsides is unfortunately not what one finds when examining the relationship between the longitude of the ascending node and the inclination of the dwarf Kreutz sungrazers, as is readily apparent from Figure 1 . An overwhelming majority of these objects in the plot is distributed along an arc that passes through the location occupied by $\mathrm{C} / 1843$ D1 and makes a sizable angle with the curve of reference apsidal orientation. Smaller numbers of the dwarf Kreutz sungrazers are also distributed along parallel arcs passing through the locations of C/1970 K1 and C/2011 W3, but much less so through $\mathrm{C} / 1965 \mathrm{~S} 1$. 
Similarly, in Figure 2 we plot the longitude of the ascending node against the argument of perihelion for the same set of the dwarf Kreutz sungrazers. This time, the dwarf and the major members appear to be distributed along an essentially common curve.

We thus find that for some reason the orbital distribution of the dwarf sungrazers differs significantly from the distribution of the major members of the Kreutz system in the plot of the longitude of the ascending node $\Omega$ against the inclination $i$ but not against the argument of perihelion $\omega$. The roots of the inconsistence between the behavior of the major and the dwarf sungrazers in Figure 1 are apparent from Figure 3 in a plot of the orientation of the apsidal line of the dwarf comets' against the position of their nodal line, that is, of $L_{\pi}$ and $B_{\pi}$ against $\Omega$. Except for the scatter, the longitude $L_{\pi}$ is seen to be

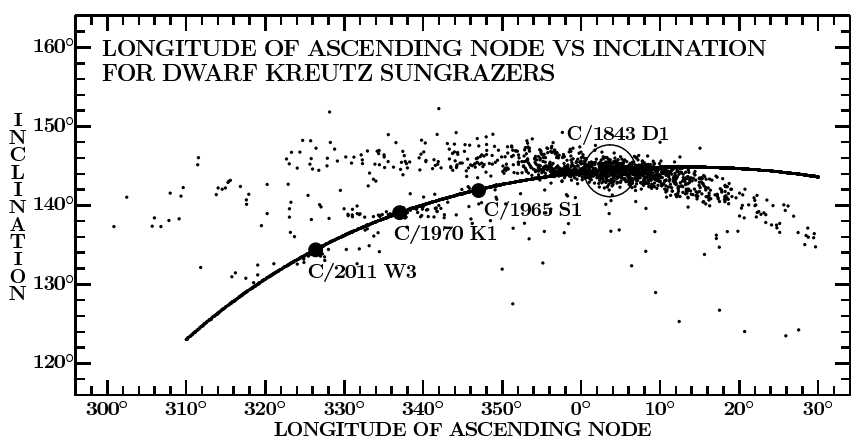

Figure 1. Plot of the longitude of the ascending node against the orbit inclination for 1565 dwarf Kreutz sungrazers from the period 1996 January to 2010 June. Also plotted are four major members of the Kreutz system: C/1843 D1 (in the center of the oversized open circle), C/1965 S1, C/1970 K1, and C/2011 W3. The solid curve is the line of reference apsidal orientation, described by $\left\langle L_{\pi}\right\rangle=282^{\circ} .8$ and $\left\langle B_{\pi}\right\rangle=+35^{\circ} .2$ (eq. J2000).

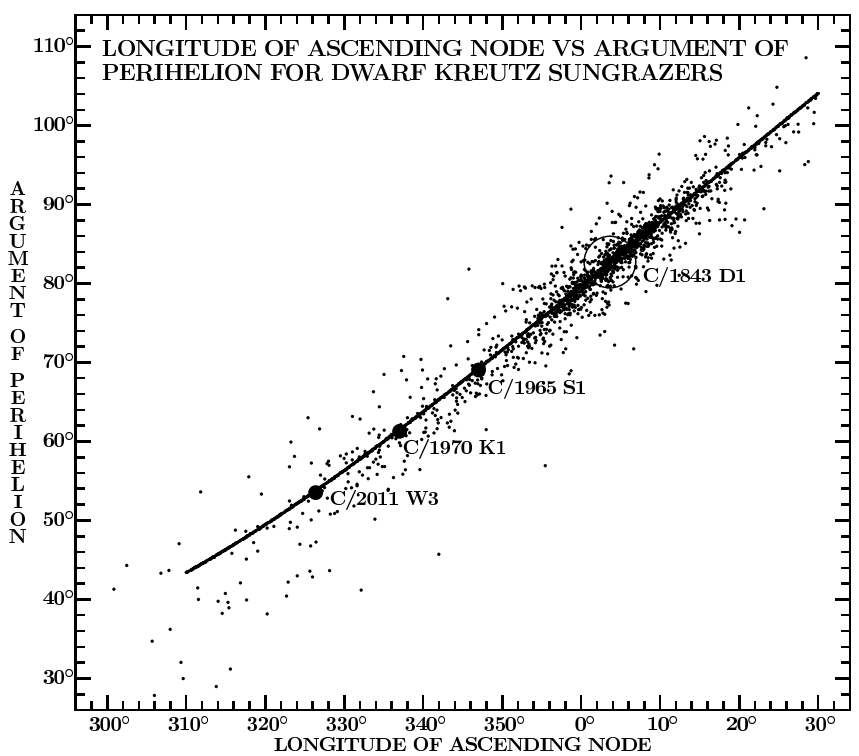

Figure 2. Plot of the longitude of the ascending node against the argument of perihelion for 1565 dwarf Kreutz sungrazers from the period 1996 January to 2010 June. Also plotted are the four major members of the Kreutz system, as in Figure 1. The solid curve is again the line of reference apsidal orientation (see the caption to Figure 1). essentially constant over a span of nearly $90^{\circ}$ in $\Omega$. In sharp contrast, the latitude $B_{\pi}$ increases systematically with $\Omega$. In fact, this trend has an effect on the plot in Figure 2 as well, but - as explained in Sec. 4, only a minor one. In addition to the thickly populated branch, which fits the position of comet C/1843 D1, we recognize in Figure 3, just as in Figure 1, the two thinly populated branches that pass, respectively, through the positions of C/1970 K1 and C/2011 W3.

By dividing the entire range of the longitudes of the ascending node into a number of intervals, the same effect is displayed in Table 2 for the thickly populated branch of the dwarf Kreutz sungrazers and in Table 3 for the other two branches. Averaging the values of $L_{\pi}$ in the penultimate column of Table 2 yields $282^{\circ} .8 \pm 0^{\circ} .8$, in perfect agreement with the adopted value of $\left\langle L_{\pi}\right\rangle$ based on the orbits of the major Kreutz-system members, while the values of $B_{\pi}$ increase systematically by nearly $25^{\circ}$ !

In order to separate the three branches from one another when calculating the mean values of the elements within each interval of $\Omega$, it was necessary also to restrict the range of corresponding inclinations. Once this was done, it was advisable also to limit $\omega$ to a certain range of values, even though Figure 2 provides no obvious clue as to where to draw the boundaries. In practice we followed a simple rule: we first incorporated all dwarf Kreutz sungrazers picked up by the computer code in the given range of $\Omega$; next we inspected the inclinations of all these entries and eliminated those (if any) judged to be clearly out of acceptable bounds; and finally we checked the arguments of perihelion of the remaining sungrazers and again removed those that appeared to be out of bounds to get the final set for each interval of $\Omega$. Admittedly, this sort of approach is always somewhat arbitrary, but only at the periphery of each set.

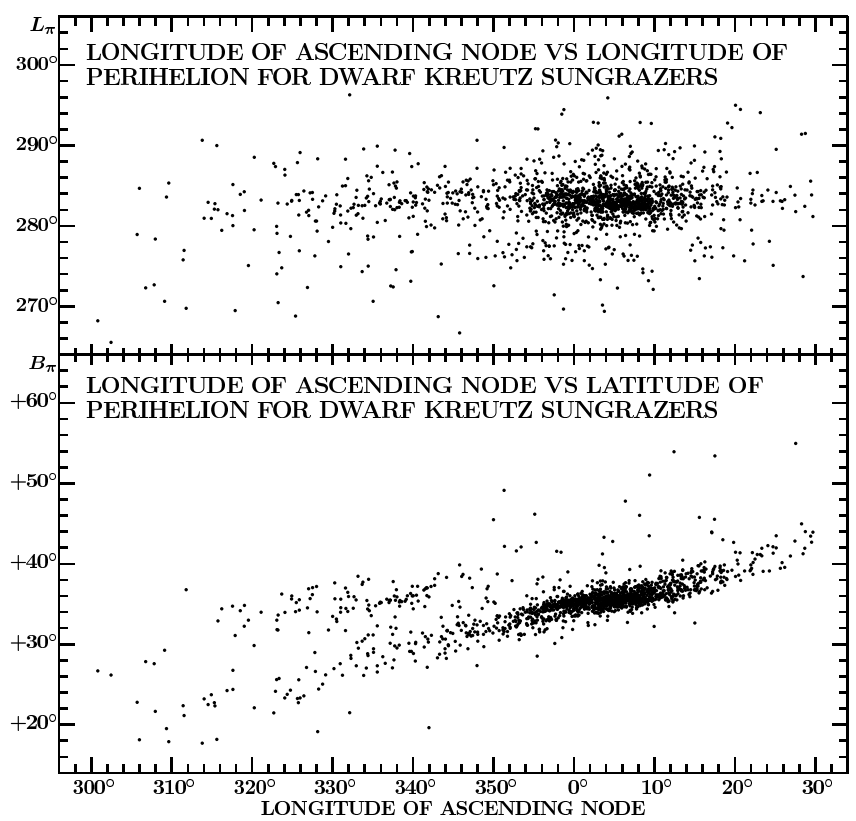

Figure 3. Plot of the longitude $L_{\pi}$ (at the top) and latitude $B_{\pi}$ of perihelion for 1565 dwarf Kreutz sungrazers from the period 1996 January to 2010 June. While the longitude stays constant over an interval of nearly $90^{\circ}$ in the nodal longitude, the latitude increases systematically, for both the thickly populated branch and the other two branches. 
Table 2

Mean Orbital Elements $\omega, \Omega, i, q$, and Longitude and Latitude of Perihelion (Eq. 2000): Branch of Dwarf Kreutz Sungrazers Fitting Orbit of Comet C/1843 D1.

\begin{tabular}{|c|c|c|c|c|c|c|c|}
\hline \multirow{2}{*}{$\begin{array}{l}\text { Interval of } \\
\text { ascending } \\
\text { nodes, } \Omega\end{array}$} & \multirow{2}{*}{$\begin{array}{c}\text { Number } \\
\text { of sun- } \\
\text { grazers }\end{array}$} & \multicolumn{4}{|c|}{ Mean value of element ${ }^{\mathrm{a}}$} & \multicolumn{2}{|c|}{ Line of apsides } \\
\hline & & $\omega$ & $\Omega$ & $i$ & $q\left(R_{\odot}\right)^{\mathrm{b}}$ & $L_{\pi}$ & $B_{\pi}$ \\
\hline $300^{\circ}-310^{\circ}$ & 5 & $32^{\circ} .1 \pm 3^{\circ} .4$ & $307^{\circ} .7 \pm 1.8$ & $140^{\circ} .0 \pm 22^{\circ} .1$ & $1.40 \pm 0.31$ & $282^{\circ} .1$ & $+19^{\circ} .9$ \\
\hline $310-320$ & 7 & $39.8 \pm 1.2$ & $315.5 \pm 1.3$ & $142.0 \pm 1.0$ & $1.28 \pm 0.18$ & 282.2 & +23.2 \\
\hline $320-330$ & 15 & $47.8 \pm 3.5$ & $325.7 \pm 2.0$ & $145.9 \pm 1.6$ & $1.35 \pm 0.27$ & 283.3 & +24.5 \\
\hline $330-335$ & 13 & $56.4 \pm 2.5$ & $332.8 \pm 1.4$ & $145.2 \pm 1.6$ & $1.35 \pm 0.34$ & 281.8 & +28.4 \\
\hline $335-340$ & 14 & $59.0 \pm 2.4$ & $337.2 \pm 1.4$ & $145.6 \pm 1.4$ & $1.27 \pm 0.22$ & 283.3 & +28.9 \\
\hline $340-345$ & 28 & $63.6 \pm 1.9$ & $342.9 \pm 1.5$ & $146.0 \pm 1.3$ & $1.20 \pm 0.20$ & 283.8 & +30.1 \\
\hline $345-350$ & 37 & $68.5 \pm 2.3$ & $347.3 \pm 1.3$ & $145.7 \pm 1.3$ & $1.21 \pm 0.20$ & 282.8 & +31.6 \\
\hline $350-355$ & 65 & $73.3 \pm 1.8$ & $353.0 \pm 1.4$ & $145.3 \pm 1.1$ & $1.18 \pm 0.20$ & 283.0 & +33.0 \\
\hline $355-\quad 0$ & 178 & $77.9 \pm 1.4$ & $358.1 \pm 1.3$ & $144.6 \pm 0.9$ & $1.12 \pm 0.13$ & 282.8 & +34.5 \\
\hline $0-\quad 5$ & 250 & $81.6 \pm 1.4$ & $2.6 \pm 1.4$ & $144.4 \pm 0.7$ & $1.12 \pm 0.13$ & 282.9 & +35.2 \\
\hline $5-10$ & 265 & $85.5 \pm 1.2$ & $7.3 \pm 1.4$ & $144.3 \pm 0.6$ & $1.15 \pm 0.16$ & 282.8 & +35.6 \\
\hline $10-15$ & 130 & $89.1 \pm 1.4$ & $12.2 \pm 1.3$ & $143.0 \pm 1.2$ & $1.19 \pm 0.19$ & 283.4 & +37.0 \\
\hline $15-20$ & 50 & $92.3 \pm 1.6$ & $16.8 \pm 1.3$ & $141.8 \pm 1.1$ & $1.17 \pm 0.19$ & 283.8 & +38.1 \\
\hline $20-30$ & 7 & $102.9 \pm 3.0$ & $28.3 \pm 1.7$ & $136.2 \pm 0.8$ & $1.18 \pm 0.20$ & 280.7 & +42.4 \\
\hline $30-40$ & 12 & $105.0 \pm 3.8$ & $34.7 \pm 2.4$ & $133.7 \pm 1.7$ & $1.27 \pm 0.29$ & 283.5 & +44.3 \\
\hline
\end{tabular}

a Samples also subjected to some limitation of intervals in $\omega$ and $i$.

b The solar radius, $R_{\odot}$, is equivalent to $0.0046548 \mathrm{AU}$.

While the two sets in Table 3 substantially support the results based on the data presented in Table 2 , there are some differences. These are likely to be due in part to the fact that the sets in Table 3 are much smaller and the errors often (though not always) larger. The average values of the angular elements are in both sets again quite close to the values for the respective major comets, but the perihelion distance of the dwarf Kreutz sungrazers in the branch fitting $\mathrm{C} / 1970 \mathrm{~K} 1$ is by more than $1 \sigma$ lesser than the comet's distance and the opposite is true for the sungrazers in the branch fitting $\mathrm{C} / 2011 \mathrm{~W} 3$. Because of the large uncertainties in the perihelion distance (which may be greatly underestimated by the formal errors calculated from the scatter among the individual objects), these discrepancies may not be significant.

Tables 2 and 3 and Figure 3 show some differences between the three branches of the dwarf Kreutz sungrazers in the rate of $\Delta B_{\pi} / \Delta \Omega$. On the average, the rate is $\left\langle\Delta B_{\pi} / \Delta \Omega\right\rangle=+0.28$ for the set in Table 2 , but +0.23 and +0.19 , respectively, for the two sets in Table 3 . The validity of this comparison may be questioned because the overall ranges of the longitude of the ascending node for the three sets are very different. However, in the intervals of $\Omega$ covered by the two sets in Table 3 , the thickly populated branch offers for $\left\langle\Delta B_{\pi} / \Delta \Omega\right\rangle$ even higher values than is the average, +0.33 and +0.30 , respectively. Thus, this effect appears to be genuine. Purely empirically, one can argue that in a clockwise rotation in Figure 1, starting from the line of reference apsidal orientation toward the thickly populated branch of sungrazers, the rate $\Delta B_{\pi} / \Delta \Omega$ keeps increasing systematically from zero to a maximum. Because the two sparsely populated branches are located in between these two, their rates are intermediate between zero and the maximum. In the lower panel of Figure 3 one can imagine a line parallel to the axis of abscissae having an ordinate of $+35^{\circ} .2$ and populated by the major sungrazers. Thus, each of the three branches, along which the overwhelming majority of the dwarf Kreutz sungrazers is distributed in Figure 1, will coincide with the line of reference apsidal orienta- tion if they are rotated counterclockwise around the respective major sungrazer's (C/1843 D1, C/1970 K1, or $C / 2011$ W3) position by the same angle, about $15^{\circ}$. This is equivalent to a rotation of the dwarf sungrazers' orbital planes by an $\Omega$-dependent amount, needed to eliminate the systematic trends in the latitude of perihelion $B_{\pi}$.

To show how well this simple-minded rotation works, Figure 4 displays the same plot of the dwarf Kreutz sungrazers as Figure 1, except that the line of reference apsidal orientation is now rotated clockwise by $15^{\circ}$ and forced to pass, respectively, through the location in the plot of comet C/1843 D1 (top branch; curve $A$ ), through the location of $\mathrm{C} / 1970 \mathrm{~K} 1$ (middle branch; curve $B$ ), and through a point close to the location of $\mathrm{C} / 2011$ W3 (lowest branch; curve $C$ ).

To summarize, the systematic trend in the perihelion latitude of the dwarf sungrazers appears to be linked to some effects that the bright members of the Kreutz system manage to avoid. And because the dwarf sungrazers

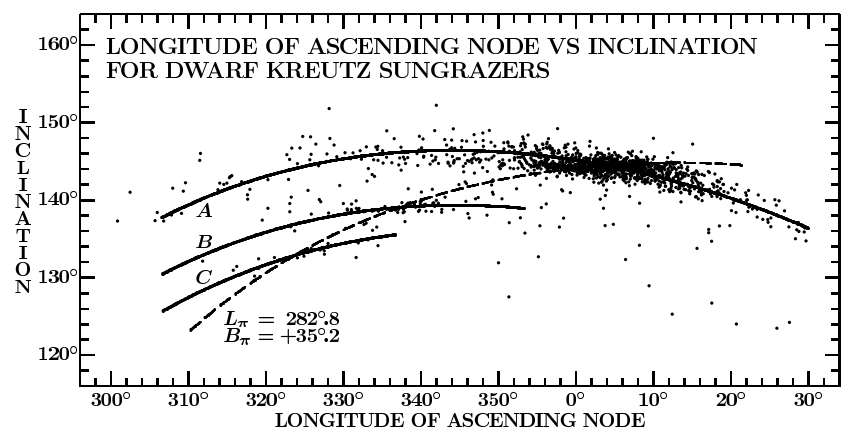

Figure 4. Plot of the longitude of the ascending node against the orbit inclination, as in Figure 1, with the three branches of the dwarf Kreutz sungrazers (dots) being compared not only to the line of reference apsidal orientation (thin dashed curve) but also to its rotated versions, marked $A, B$, and $C$, that pass, respectively, through the positions of three major Kreutz sungrazers: C/1843 D1, C/1970 K1, and, approximately, C/2011 W3. The angle of rotation, $15^{\circ}$, is the same for all three populations of the dwarf sungrazers. Thus, the match, which is very satisfactory, is not coming from, and does not represent, least-squares solutions. 
Table 3

Mean Orbital Elements $\omega, \Omega, i, q$, and Longitude and Latitude of Perihelion (Eq. 2000): Branches of Dwarf Kreutz Sungrazers Fitting Orbits of C/1970 K1 or C/2011 W3.

\begin{tabular}{|c|c|c|c|c|c|c|c|}
\hline \multirow{2}{*}{$\begin{array}{c}\text { Interval of } \\
\text { ascending } \\
\text { nodes, } \Omega\end{array}$} & \multirow{2}{*}{$\begin{array}{l}\text { Number } \\
\text { of sun- } \\
\text { grazers }\end{array}$} & \multicolumn{4}{|c|}{ Mean value of element ${ }^{a}$} & \multicolumn{2}{|c|}{ Line of apsides } \\
\hline & & $\omega$ & $\Omega$ & $i$ & $q\left(R_{\odot}\right)$ & $L_{\pi}$ & $B_{\pi}$ \\
\hline \multicolumn{8}{|c|}{ Branch Fitting C/1970 K1 } \\
\hline $320^{\circ}-330^{\circ}$ & 6 & $53^{\circ} .0 \pm 33^{\circ} .4$ & $324^{\circ} .1 \pm 2^{\circ} .9$ & $138^{\circ} .5 \pm 1.2$ & $1.49 \pm 0.20$ & $279^{\circ} .3$ & +31.9 \\
\hline $330-335$ & 20 & $58.5 \pm 2.8$ & $332.3 \pm 1.6$ & $138.4 \pm 1.0$ & $1.59 \pm 0.26$ & 281.7 & +34.5 \\
\hline $335-340$ & 27 & $62.0 \pm 3.2$ & $337.7 \pm 1.4$ & $139.0 \pm 0.6$ & $1.63 \pm 0.22$ & 282.9 & +35.4 \\
\hline $340-345$ & 18 & $66.6 \pm 3.6$ & $341.8 \pm 1.3$ & $139.6 \pm 1.0$ & $1.51 \pm 0.29$ & 281.4 & +36.5 \\
\hline $345-355$ & 14 & $72.0 \pm 3.9$ & $348.5 \pm 2.3$ & $140.2 \pm 1.8$ & $1.44 \pm 0.29$ & 281.4 & +37.5 \\
\hline \multicolumn{8}{|c|}{ Branch Fitting C/2011 W3 } \\
\hline $315^{\circ}-320^{\circ}$ & 6 & $47^{\circ} .5 \pm 1^{\circ} .5$ & $317^{\circ} .7 \pm 1^{\circ} .4$ & $131^{\circ} .1 \pm 0^{\circ} .7$ & $1.37 \pm 0.28$ & $282^{\circ} .0$ & $+33^{\circ} .8$ \\
\hline $320-325$ & 4 & $50.9 \pm 2.1$ & $323.7 \pm 1.8$ & $132.7 \pm 0.3$ & $1.45 \pm 0.31$ & 283.9 & +34.8 \\
\hline $325-330$ & 7 & $54.5 \pm 1.7$ & $326.9 \pm 0.9$ & $133.6 \pm 0.4$ & $1.48 \pm 0.18$ & 282.9 & +36.2 \\
\hline $330-335$ & 9 & $58.5 \pm 1.8$ & $332.6 \pm 1.6$ & $135.5 \pm 1.2$ & $1.48 \pm 0.33$ & 283.3 & +36.7 \\
\hline
\end{tabular}

${ }^{\text {a }}$ Samples also subjected to some limitation of intervals in $\omega$ and $i$.

could not, as individual bodies, survive longer than one revolution about the Sun (otherwise they should be seen to recede from the Sun after perihelion), the effects in Figures 1 and 3 and in Tables 2 and 3 should be a product of the dwarf sungrazers' evolution in the course of this revolution about the Sun.

Tables 2 and 3 also present the averaged perihelion distance $q$ of the dwarf sungrazers in each interval of the longitude of the ascending node. We find that in the thickly populated branch the minimum nominal distance of $\sim 1.1 R_{\odot}$ is reached near $\Omega \simeq 0^{\circ}$ but that the minima are closer to $1.4-1.5 R_{\odot}$ in the two thinly populated branches. Because of the uncertainties involved and also because the orbits of some of these objects were derived with the perihelion distance being forced by Marsden to particular values (Sekanina 2002; see also Sec. 9), one should not attach much significance to these variations.

A more interesting result is found from inspection of the entries for the interval of $0^{\circ}<\Omega<5^{\circ}$ in Table 2. All five tabulated angles equal, within the limits of error, the respective angular elements of comet C/1843 D1 (Table 1 and Sekanina \& Chodas 2008). This match strongly suggests a close relationship between this comet and the most populated branch of the dwarf Kreutz sungrazers despite the $150+$ years spanned between them.

The contrast between the plot of $i$ against $\Omega$ (Figure 1 ), in which the dwarf Kreutz sungrazers behave differently from the major members, and the plot of $\omega$ against $\Omega$, in which the two categories of objects almost overlap, is illustrated best by the calculated values of the elements at the ends of the $\Omega$ range in Table 2 . At $\Omega=307^{\circ} .7$ the difference between the major and the dwarf members is nearly $-20^{\circ}$ in $i$ but only $+10^{\circ}$ in $\omega$, while at $\Omega=34^{\circ} .7$ it is $+9^{\circ}$ in $i$ but about $-3^{\circ}$ in $\omega$. We next address these discrepancies, as well as the differences in the behavior of $L_{\pi}$ and $B_{\pi}$ of the dwarf Kreutz sungrazers in Figure 3 and Tables 2 and 3, in terms of perturbations caused by momentum changes in the dwarf sungrazers' motions.

\section{PERTURBATIONS DUE TO MOMENTUM CHANGES IN THE ORBITAL MOTIONS OF THE DWARF KREUTZ SUNGRAZERS}

The enormous population of the dwarf Kreutz sungrazers could have arisen on the timescale of a single revo- lution about the Sun only by cascading fragmentation of larger objects in the Kreutz system, which are likely to have included some very massive members. One can hypothesize that, as a consequence of numerous fragmentation events, the orbits of the fragments have gradually been modified compared to the initial parent's orbit, and that their observed distribution reflects a cumulative effect of these modifications. If so, the question then arises about the nature of these effects that (a) show up in the plot of $\Omega$ against $i$, but not (at least nowhere as prominently) in the plot of $\Omega$ against $\omega$, and (b) manifest themselves as systematic variations in the perihelion latitude $B_{\pi}$ but not in the longitude $L_{\pi}$.

Aiming to examine the nature of the effect in Figures 1 and 3 and in Tables 2 and 3, we begin with the relations between (a) the instantaneous rate of change, at time $t$, of the angular orbital elements - $d \omega / d t, d \Omega / d t, d i / d t$ - and (b) the momentum acquired during a breakup, expressed in terms of the components of an acceleration $j(t)$ imparted to a fragment relative to the parent body in the three cardinal directions defined by the heliocentric orbit of the parent in the radial (away from the Sun), $\mathrm{R}$, transverse, $\mathrm{T}$, and normal, $\mathrm{N}$, directions of the righthanded RTN coordinate system. These relations can be written thus (e.g., Danby 1988):

$$
\left(\begin{array}{c}
\frac{d \omega}{d t} \\
\frac{d \Omega}{d t} \\
\frac{d i}{d t}
\end{array}\right)=\left(\begin{array}{ccc}
X_{\mathrm{R}} & X_{\mathrm{T}} & X_{\mathrm{N}} \\
Y_{\mathrm{R}} & Y_{\mathrm{T}} & Y_{\mathrm{N}} \\
Z_{\mathrm{R}} & Z_{\mathrm{T}} & Z_{\mathrm{N}}
\end{array}\right) \cdot\left(\begin{array}{c}
j_{\mathrm{R}} \\
j_{\mathrm{T}} \\
j_{\mathrm{N}}
\end{array}\right)
$$

where

$$
\begin{aligned}
& X_{\mathrm{R}}=\frac{\chi}{e^{2}}(1-\psi), \\
& X_{\mathrm{T}}=\frac{\chi}{e \psi}(1+\psi) \sin u, \\
& X_{\mathrm{N}}=-\frac{\chi}{\psi} \cot i \sin (\omega+u), \\
& Y_{\mathrm{N}}=\frac{\chi}{\psi} \frac{\sin (\omega+u)}{\sin i}, \\
& Z_{\mathrm{N}}=\frac{\chi}{\psi} \cos (\omega+u),
\end{aligned}
$$


and

$$
Y_{\mathrm{R}}=Y_{\mathrm{T}}=Z_{\mathrm{R}}=Z_{\mathrm{T}}=0,
$$

with $\chi=\sqrt{p} / k, \psi=p / r, k$ being the Gaussian gravitational constant, $p$ the parameter of the orbit, $p=q(1+e)$, $q$ the perihelion distance, $e$ the eccentricity of the orbit, and $r=r(t)$ and $u=u(t)$, respectively, the heliocentric distance and the true anomaly at time $t$.

Since the fragmentation process is assumed to consist of discrete, short-term events, of a duration $\Delta t \rightarrow 0$ and our interest is only in their integrated outcome, we replace the derivative $d \omega / d t$ on the left-hand side of Eq. (3) with

$$
\Delta \omega=\int_{(\Delta t \rightarrow 0)} \frac{d \omega(t)}{d t} d t
$$

and similarly $\Delta \Omega$ and $\Delta i$; and, on the right-hand side, we introduce the components of the separation velocity,

$$
V_{\mathrm{R}}=\int_{(\Delta t \rightarrow 0)} j_{\mathrm{R}}(t) d t
$$

and similarly $V_{\mathrm{T}}$ and $V_{\mathrm{N}}$. Since the events are brief, the heliocentric distance $r$ and the true anomaly $u$ are constants (i.e., $\Delta r \rightarrow 0$ and $\Delta u \rightarrow 0$ ). Equation (3) now becomes

$$
\left(\begin{array}{c}
\Delta \omega \\
\Delta \Omega \\
\Delta i
\end{array}\right)=\left(\begin{array}{ccc}
X_{\mathrm{R}} & X_{\mathrm{T}} & X_{\mathrm{N}} \\
0 & 0 & Y_{\mathrm{N}} \\
0 & 0 & Z_{\mathrm{N}}
\end{array}\right) \cdot\left(\begin{array}{c}
V_{\mathrm{R}} \\
V_{\mathrm{T}} \\
V_{\mathrm{N}}
\end{array}\right)
$$

Increments $\Delta \omega, \Delta \Omega$, and $\Delta i$ affect increments $\Delta L_{\pi}$ and $\Delta B_{\pi}$ in the direction to perihelion, which are equal to

$$
\begin{gathered}
\Delta L_{\pi}=\Delta \Omega+\frac{\cos i}{1-\sin ^{2} \omega \sin ^{2} i}\left(\Delta \omega-\frac{1}{2} \Delta i \sin 2 \omega \tan i\right), \\
\Delta B_{\pi}=\frac{\cos \omega \sin i}{\sqrt{1-\sin ^{2} \omega \sin ^{2} i}}(\Delta \omega+\Delta i \tan \omega \cot i) .
\end{gathered}
$$

To the extent that Figure 3 implies that, on the average, $\Delta L_{\pi}=0$, the following constraint on $V_{\mathrm{R}}, V_{\mathrm{T}}$, and $V_{\mathrm{N}}$ applies after inserting for $\Delta \omega, \Delta \Omega$, and $\Delta i$ from (8) and (4) into the first equation of (9):

$$
X_{\mathrm{R}} V_{\mathrm{R}}+X_{\mathrm{T}} V_{\mathrm{T}}=-\Psi \cos \omega \tan i \sin u \cdot V_{\mathrm{N}},
$$

where

$$
\Psi=\frac{\chi}{\psi}=\frac{r}{k \sqrt{p}}
$$

The second equation of (9) can similarly be rewritten as

$$
\begin{aligned}
\Delta B_{\pi}= & \frac{\cos \omega \sin i}{\sqrt{1-\sin ^{2} \omega \sin ^{2} i}} \\
& \times\left(X_{\mathrm{R}} V_{\mathrm{R}}+X_{\mathrm{T}} V_{\mathrm{T}}-\Psi \sec \omega \cot i \sin u \cdot V_{\mathrm{N}}\right) .
\end{aligned}
$$

Inserting for $X_{\mathrm{R}} V_{\mathrm{R}}+X_{\mathrm{T}} V_{\mathrm{T}}$ from (10) into (12) we get the final expression for $\Delta B_{\pi}$ :

$$
\Delta B_{\pi}=-\Psi \frac{\cos i \sin u}{\sqrt{1-\sin ^{2} \omega \sin ^{2} i}}\left(1+\cos ^{2} \omega \tan ^{2} i\right) \cdot V_{\mathrm{N}} .
$$

This equation says that as long as the perihelion longitude $L_{\pi}$ of the dwarf Kreutz sungrazers in Figure 1 is statistically independent of the three angular orbital elements, the systematic increment in the perihelion latitude is only a function of a momentum change in the direction normal to the orbital plane.

On condition (10), the increments in all three angular elements depend also only on the normal component,

$$
\begin{gathered}
\Delta \omega=-\Psi \cot i\left[\sin (\omega+u)+\cos \omega \tan ^{2} i \sin u\right] V_{\mathrm{N}}, \\
\Delta \Omega=\Psi \frac{\sin (\omega+u)}{\sin i} V_{\mathrm{N}}, \\
\Delta i=\Psi \cos (\omega+u) V_{\mathrm{N}} .
\end{gathered}
$$

The expressions for the ratio of increments of the angles plotted in Figures 1 and 2 we obtain, respectively,

$$
\frac{\Delta i}{\Delta \Omega}=\sin i \cot (\omega+u) \approx \sin i \cot \omega
$$

and

$$
\frac{\Delta \omega}{\Delta \Omega}=-\cos i\left[1+\frac{\cos \omega \tan ^{2} i \sin u}{\sin (\omega+u)}\right] \approx-\cos i,
$$

where the approximations reflect the fact that the true anomaly $u \rightarrow \pm 180^{\circ}$ everywhere in the orbit except very close to perihelion. Equations (15) and (16) can also be derived directly from Eqs. (2). Now, there is one major difference between Eq. (15) and Eq. (16). As a function of merely the inclination, which varies within fairly tight limits, mostly between $\sim 130^{\circ}$ and $\sim 150^{\circ}$ (Figure 1 ), the ratio $\Delta \omega / \Delta \Omega$ varies between +0.64 and +0.87 , which explains nearly linear relationship between the two elements with an average slope of +0.75 in Figure 2. By contrast, the ratio $\Delta i / \Delta \Omega$ depends on both the inclination and the argument of perihelion, which varies widely, from $\sim 30^{\circ}$ to $\sim 110^{\circ}$ (Figure 2 ). Hence, $\Delta i / \Delta \Omega$ can be of either sign and the curve of reference apsidal orientation in the plot of $i=i(\Omega)$ reaches a maximum at $\omega=90^{\circ}$, or $\Omega=12^{\circ} .8$, when $i=180^{\circ}-B_{\pi}=144.8$ from Eq. (2).

\section{INTERPRETING THE VARIATIONS IN THE LATITUDE OF PERIHELION}

Analysis in Sec. 4 of the variations in the angular orbital elements and in the orientation of the line of apsides of the dwarf Kreutz sungrazers' orbits has shown that the observed systematic increase in the latitude of perihelion with the longitude of the ascending node in Figure 3 and in Tables 2 and 3 is - on the condition of an invariable longitude of perihelion (also apparent from the figure and the two tables) - a product of some perturbations, or momentum increments, in the direction normal to the comets' orbital planes. This effect can in principle be either continuous or discrete. The magnitude of the effect is so profound that, if discrete, it must consist of a large number of individual events, because the separation velocities during a cometary splitting are known to be at most only a few meters per second (e.g., Sekanina 1982, $2005)$, much too low to fit $\Delta B_{\pi} \simeq 25^{\circ}$.

\subsection{Interpretation in Terms of a Sequence of Fragmentation Events}

The first scenario we consider is based on a hypothesis that the large range of perihelion latitudes represents an accumulation of minor impulses acquired by these dwarf comets during the many fragmentation events in the course of one revolution about the Sun, from perihelion to next perihelion. 
To scrutinize this hypothesis in some detail, we need to postulate a law governing the sequence of such fragmentation events and to estimate an average normal component of the impulse (differential velocity) a fragment ought to acquire per event in order to match the total observed effect. For this purpose we adopt the fragmentation sequence proposed by Sekanina (2002), which was found to be in fair agreement with the known sequence of secondary breakups of comet D/1993 F2 (ShoemakerLevy 9 ). The time $t_{m}$ of an $m$-th event is given by

$$
t_{m}=t_{0}+\frac{\Gamma^{m}-1}{\Gamma-1} \Delta t,
$$

where $t_{0}$ is the time of the primary fragmentation event (close to perihelion), which is tidal or tidally-supported or triggered in nature; $\Delta t$ is an initial interval for secondary fragmentation; and $\Gamma>1$ is a dimensionless constant that describes the rate of fragmentation slowdown along the orbit. If we reckon time from perihelion, then $t_{0} \simeq 0$ and $\nu_{0}$, the total number of fragmentation events or impulses imparted to a fragment, equals

$$
\nu_{0}=\frac{\log \left[1+(\Gamma-1) P_{\text {orb }} / \Delta t\right]}{\log \Gamma},
$$

where $P_{\text {orb }}$ is the orbital period. This equation is a relation between $\nu_{0}, \Gamma$, and $\Delta t$. If, as was argued above, the dwarf Kreutz sungrazers in the thickly populated branch in Figure 1 are closely related to comet C/1843 D1 and if this comet, as postulated in Sekanina \& Chodas (2007), is indeed the most massive known fragment of the celebrated sungrazer X/1106 C1, then the dwarf Kreutz sungrazers have orbital periods close to $P_{\text {orb }}=900$ years.

Next, it is necessary to adopt a certain breakup pattern. Let us first consider that a Kreutz fragment born from the primary breakup at the Sun splits into two approximately equal pieces, each of which again breaks up into two about equal parts, etc., until the fragments are eventually as small as the faint dwarf sungrazers. Assuming $10^{18}$ grams for the mass $\mathcal{M}_{\text {init }}$ of the initial fragment and $10^{6}$ grams for an average mass $\mathcal{M}_{\text {fin }}$ of the final products, the number of fragmentation events $\nu_{0}$ must satisfy a condition $\mathcal{M}_{\text {fin }}=2^{-\nu_{0}} \mathcal{M}_{\text {init }}$, so that in this case $\nu_{0} \simeq 40$. Equation (18) is now a relation between $\Gamma$ and $\Delta t$. For example, for $\Gamma$ between 1.1 and 1.2 the initial interval $\Delta t$ drops from 2 years to 45 days.

It is now possible to apply Equation (13) to the proposed sequence of fragmentation events, described by pairs of various values of $\Gamma$ and $\Delta t$. Expressing $\Delta B_{\pi}$ in degrees, $p$ and $r$ in $\mathrm{AU}$, and $V_{\mathrm{N}}$ in $\mathrm{m} \mathrm{s}^{-1}$, the reciprocal gravitational constant $1 / k=0^{\circ} .001924$. The product $|r \sin u|$, the variable in Eq. (13) for $\Delta B_{\pi}$, reaches a peak value

$$
|r \sin u|_{\text {peak }}=q(1+e)^{\frac{1}{2}}(1-e)^{-\frac{1}{2}}=a_{0}\left(1-e^{2}\right)^{\frac{1}{2}}
$$

at a distance $r_{\text {peak }}$ that is equal to the semimajor axis $a_{0}$ of the orbit. Adopting $q \simeq 0.0055 \mathrm{AU}$ and $e \simeq 0.99994$, or $a_{0}=91.7 \mathrm{AU}$, we find $|r \sin u|_{\text {peak }} \simeq 1.00 \mathrm{AU}$ and for any single event $\left|\Delta B_{\pi}\right| \ll 0^{\circ} .04 V_{\mathrm{N}}$.

Since the normal component of the separation velocity of fragments of the split comets does not exceed a few meters per second (see the first paragraph of Sec. 5) and, in addition, the sign of the expression for $\Delta B_{\pi}$ changes at aphelion (because of $\sin u$ ), so that $\Sigma \Delta B_{\pi} \ll \Sigma\left|\Delta B_{\pi}\right|$, we conclude that this scenario cannot explain the magnitude of the systematic rate of change in the latitude of perihelion with the longitude of the ascending node in Tables 2-3 and Figure 3. This holds true even if we adopt a different breakup pattern with a much greater total number of fragmentation events and/or a different law governing their sequence.

This information is consistent with the perturbations due to a fragment's separation velocity, listed in Table 8 of Sekanina (2002). While a normal velocity of $5 \mathrm{~ms}^{-1}$ can trigger a change of up to nearly $27^{\circ}$ in the longitude of the nodal line and up to nearly $5^{\circ}$ in the inclination, it fails to shift the latitude of perihelion by even $0^{\circ} .1$.

\subsection{Interpretation in Terms of Perturbations by a Major Nongravitational Force Near the Sun}

This category of hypotheses regards sizable deviations of the lines of apsides of dwarf Kreutz sungrazers from the line of reference apsidal orientation (Figure 3 and Tables 2 and 3 ) to be an effect of a continuous force, which is nongravitational in nature and acts while these sungrazers are under observation. We first discuss briefly the Lorentz force, which acts in the direction normal to the orbital plane.

The Lorentz force is known to appreciably affect the motions of submicron-sized charged particles of dust. Compelling evidence suggests, however, that the dust tails of the dwarf Kreutz sungrazers, consisting of such microscopic grains, are subjected to no detectable effects of the Lorentz force (Sekanina 2000, Thompson 2009). On the strength of this argument, it is inconceivable that the nuclei of the dwarf Kreutz sungrazers could be subjected to this force to an extent of triggering a major effect of the kind examined.

An alternative scenario is based on an assumption that these are effects due to a momentum transferred to the nucleus by sublimation of water ice and/or more refractory species at small heliocentric distances, at which the dwarf Kreutz sungrazers are observed. Such small heliocentric distances are strongly suggested by a peak inclination near $\Omega \simeq 340^{\circ}$ in Figure 1 . This value of $\Omega$ corresponds to $\omega \simeq 63^{\circ}$ in Figure 2. Furthermore, the peak inclination requires that $\cot (\omega+u)=0$ in Eq. (15), so that $u \simeq-153^{\circ}$, equivalent to a time a little less than 1 day before perihelion, when the dwarf sungrazers are indeed observed.

In order for this scenario to work - that is, to explain the effect normal to the orbital plane - we need to test whether the introduction into the equations of motion of a term containing a normal component of the momentum-transfer acceleration could offer orbital solutions in which the angular elements are consistent with the reference apsidal orientation presented in Table 1.

The standard orbit determination technique, which was developed by Marsden et al. (1973) and whose versions are nowadays almost universally employed worldwide to compute cometary orbits, sets up the nongravitational terms in the three cardinal directions of an RTN right-handed coordinate system, ${ }^{2}$ identical to that in Sec. 4. The introduced law, $g_{\text {ice }}(r)$ (see Sec. 7), mimicks the dependence of an averaged water-ice sublimation rate on heliocentric distance $r$. The magnitude of the

\footnotetext{
${ }^{2}$ RTN $=$ Radial $/$ Transverse/Normal.
} 
momentum-transfer acceleration at $1 \mathrm{AU}$ from the Sun is measured by the so-called Style II parameters, $A_{1}$ in the radial direction (away from the Sun), $A_{2}$ in the transverse direction in the orbital plane, and $A_{3}$ in the direction normal to the orbital plane (Marsden et al. 1973). Their values are tabulated in units of $10^{-8} \mathrm{AU}_{\text {day }}{ }^{-2}$, sometimes without an explicitly listed exponent. ${ }^{3}$ For practically all comets whose motions have required the incorporation of the nongravitational terms into the equations of motion, successful orbital solutions have almost always been obtained by ignoring the acceleration's normal component $\left(A_{3}=0\right)$. Marsden \& Williams' (2008) catalogue of orbits does not even have a column for $A_{3}$.

To examine the proposed scenario, we first selected a small set of dwarf Kreutz sungrazers from a catalogue of about 1600 of them (Marsden \& Williams 2008; plus the objects from mid-May 2008 to mid-June 2010 ${ }^{4}$ ). Each selected dwarf sungrazer was to satisfy these conditions: (i) be a member of the thickly populated branch in Figure 1 ; (ii) the value of $\Omega$ to come from a broad range of the longitudes of the ascending node, between $\sim 300^{\circ}$ and $\sim 40^{\circ}$ (Table 2); (iii) as published, the parabolic orbit be consistent with a least-squares solution, with no element forced to any particular value; and (iv) the orbit be based on at least seven astrometric positions, measured from the images taken with the $\mathrm{C} 2$ coronagraph on board the $S O H O$ satellite and/or with one of the COR2 coronagraphs on board the two STEREO spacecraft. This last condition stems from our focus on the final segment of the trajectory and from our need to have astrometric observations of the best possible quality. Because of the pixel sizes, $11^{\prime \prime} .4$ for the C2 coronagraph and $14^{\prime \prime} .7$ for the COR2 coronagraphs, more accurate data were expected from them than from the wide-field coronagraphs, C3 $\left(56^{\prime \prime} / \mathrm{pixel}\right)$ and HI1 (70"/ pixel). However, the pixel-size advantage of $\mathrm{C} 2$ may sometimes be offset by scarcity or a very uneven distribution of reference stars over the image field.

All orbital computations were carried out by the second author, who employed a code EXORB 7 developed by A. Vitagliano. The code includes the perturbations by the eight major planets, Pluto, and the three most massive asteroids. It employs the standard DE406 library and allows one to use a forced value for any orbital element or nongravitational parameter, an option that was copiously exploited. For each of the selected dwarf Kreutz sungrazers, two sets of orbital elements were derived. The first set was a parabolic gravitational solution, whereas the second set was a restricted nongravitational solution, which employed a parabolic approximation and Marsden et al.'s (1973) standard formalism, in which we assumed that $A_{1}=A_{2}=0$ and which we used to search for the magnitude of the acceleration component normal to the orbit plane, $A_{3} \neq 0$. Because of fairly large uncertainties in the astrometric observations and very short orbital arcs observed, we decided at this point not to incorporate $A_{3}$ as a variable directly into the least-squares differential optimization procedure, but, instead, to proceed by iteration.

\footnotetext{
${ }^{3}$ A unit of $10^{-8} \mathrm{AU}$ day ${ }^{-2}=2.004 \times 10^{-5} \mathrm{~cm} \mathrm{~s}^{-2}$; at $1 \mathrm{AU}$ from the Sun it equals $3.38 \times 10^{-5}$ the Sun's gravitational acceleration.

${ }^{4}$ These orbital elements have been published in numerous MPCs starting with MPC 63377 and ending with MPC 72855.
}

Based on our estimates of several meters for the nuclear sizes of faint dwarf Kreutz sungrazers near the end of their lifetime (by extrapolating the derived diameters of brighter ones; e.g., Sekanina 2003) and on the highest known values of $A_{1} \sim 20-30 \times 10^{-8} \mathrm{AU}_{\text {day }^{-2}}$ (Marsden \& Williams 2008) among ordinary long-period comets (in particular, objects such as C/1993 A1 or C/1998 P1) with presumably kilometer- or subkilometersized nuclei, we began each computer run with an initial value of $A_{3} \approx 10^{-5} \mathrm{AU}_{\mathrm{day}^{-2}}$. This was a fairly conservative estimate since the comparison comets, of perihelion distances comparable to or exceeding 1 AU, imply momentum-transfer effects due almost exclusively to water ice driven sublimation, while at the heliocentric distances at which the dwarf Kreutz sungrazers are observed - around $0.05 \mathrm{AU}$ or $\sim 11 R_{\odot}$ - numerous species less volatile than water ice also sublimate profusely. Together with progressive fragmentation this should increase the magnitude of the momentum-transfer acceleration. Thus, applying a least-squares optimization procedure to derive the orbital elements, without removing any of the astrometric positions available, we searched for a value of $A_{3}$ that, in the context of the common origin of the Kreutz sungrazers (Sec. 1), provided a minimum offset from the reference apsidal orientation.

The results for eight test dwarf Kreutz sungrazers are summarized in Table 4, in which we compare three sets of orbital solutions. The first set, in the row Cat., is the orbit as computed by Marsden; it is copied either from Marsden \& Williams' (2008) catalogue (entries 1-3 and 6), or from MPC 63599-63601 (entries 4 and 7-8) or MPC 66704 (entry 5).

The second set, in the row Grav., represents our own parabolic gravitational approximation. We made this run in order to confirm that Marsden's results, the details of which have never been published, are closely reproduced. Comparison with the catalogued orbital elements tolerated formal differences of up to a few tenths of a degree in the angular elements and up to $0.01 R_{\odot}$ in the perihelion distance. As expected, the perihelion times always agreed to better than 0.01 day. However, a number of objects, especially from the earlier times, considered initially as suitable test cases, had to be rejected, because the catalogued perihelion distances were forced by Marsden, usually upwards but sometimes downwards, to make them exceed $1 R_{\odot}$ but stay smaller than $\sim 2 R_{\odot}$. This manipulation resulted in changes in the other elements as well, including the longitude of the ascending node, and consequently interfered with our effort to have the test sungrazers distributed more or less uniformly between $\Omega \simeq 300^{\circ}$ and $\Omega \simeq 40^{\circ}$.

The third set, in the row $\left(A_{3}\right)$, is our nongravitational solution, referred to above. The deviation of an iterated value of $A_{3}$ from the value we were searching for was measured by an offset of the iterated apsidal orientation, given by the perihelion coordinates $L_{\pi}$ and $B_{\pi}$,

$$
\begin{aligned}
\tan \left(L_{\pi}-\Omega\right) & =\tan \omega \cos i \\
\sin B_{\pi} & =\sin \omega \sin i
\end{aligned}
$$

from the reference orientation. The offset $\epsilon$, whose minimum we were aiming at, was computed from

$$
\cos \epsilon=\sin B_{\pi} \sin \left\langle B_{\pi}\right\rangle+\cos B_{\pi} \cos \left\langle B_{\pi}\right\rangle \cos \left(L_{\pi}-\left\langle L_{\pi}\right\rangle\right) .
$$


Nongravitational Motions of Dwarf Kreutz Sungrazers

Table 4

Comparison of Gravitational Solutions for Eight Test Dwarf Kreutz Sungrazers with Their Nongravitational Solutions Containing $A_{3}$.

\begin{tabular}{|c|c|c|c|c|c|c|c|c|c|c|c|c|}
\hline \multirow[b]{2}{*}{ No. } & \multirow[b]{2}{*}{ Object } & \multirow{2}{*}{$\begin{array}{l}\text { Orbital } \\
\text { solution }^{\mathrm{a}}\end{array}$} & \multicolumn{5}{|c|}{ Orbital elements (eq. J2000) } & \multirow{2}{*}{$\begin{array}{l}\text { Param- } \\
\text { eter } A_{3} \\
\left(\text { units }^{b}\right)\end{array}$} & \multicolumn{2}{|c|}{ Line of apsides } & \multirow{2}{*}{$\begin{array}{l}\text { Apsidal } \\
\text { line's } \\
\text { offset }\end{array}$} & \multirow{2}{*}{$\begin{array}{l}N_{\text {obs }} \\
\text { RMS } \\
\text { residual }\end{array}$} \\
\hline & & & $t_{\pi}(\mathrm{ET})$ & $\omega$ & $\Omega$ & $i$ & $q\left(R_{\odot}\right)$ & & $L_{\pi}$ & $B_{\pi}$ & & \\
\hline \multirow[t]{3}{*}{1} & \multirow[t]{3}{*}{$\mathrm{C} / 2007 \mathrm{X} 13$} & Cat. & $2007 / 12 / 14.42$ & $27^{\circ} .73$ & $305^{\circ} .98$ & $138^{\circ} .31$ & 1.63 & & $284^{\circ} .55$ & $+18^{\circ} .03$ & $17^{\circ} .24$ & 8 \\
\hline & & Grav. & $2007 / 12 / 14.42$ & 27.76 & 306.00 & 138.31 & 1.62 & & 284.54 & +18.05 & 17.22 & $\pm 12^{\prime \prime} .7$ \\
\hline & & $\left(A_{3}\right)$ & $2007 / 12 / 14.53$ & 87.33 & 9.69 & 144.87 & 1.03 & -25.1 & 282.95 & +35.08 & 0.17 & $\pm 13^{\prime \prime} .0$ \\
\hline \multirow[t]{3}{*}{2} & \multirow[t]{3}{*}{$\mathrm{C} / 2007 \mathrm{X} 3$} & Cat. & $2007 / 12 / 05.16$ & 39.53 & 315.29 & 142.81 & 1.37 & & 281.97 & +22.63 & 12.59 & 7 \\
\hline & & Grav. & $2007 / 12 / 05.16$ & 39.55 & 315.30 & 142.81 & 1.38 & & 281.96 & +22.64 & 12.58 & $\pm 5^{\prime \prime} .6$ \\
\hline & & $\left(A_{3}\right)$ & $2007 / 12 / 05.25$ & 82.08 & 4.55 & 145.18 & 1.33 & -14.8 & 284.17 & +34.44 & 1.36 & $\pm 6^{\prime \prime} .6$ \\
\hline \multirow[t]{3}{*}{3} & \multirow[t]{3}{*}{$\mathrm{C} / 2001 \mathrm{Y} 4$} & Cat. & $2001 / 12 / 18.62$ & 42.87 & 324.05 & 144.61 & 1.50 & & 286.93 & +23.20 & 12.53 & 10 \\
\hline & & Grav. & $2001 / 12 / 18.62$ & 42.86 & 324.03 & 144.60 & 1.51 & & 286.93 & +23.20 & 12.53 & $\pm 20^{\prime \prime} .0$ \\
\hline & & $\left(A_{3}\right)$ & $2005 / 12 / 18.68$ & 86.79 & 9.22 & 144.68 & 1.02 & -4.18 & 283.15 & +35.26 & 0.29 & $\pm 20^{\prime \prime} .8$ \\
\hline \multirow[t]{3}{*}{4} & \multirow[t]{3}{*}{$\mathrm{C} / 2008 \mathrm{M} 4$} & Cat. & $2008 / 06 / 25.69$ & 58.09 & 338.00 & 144.63 & 1.29 & & 285.37 & +29.43 & 6.16 & 10 \\
\hline & & Grav. & $2008 / 06 / 25.69$ & 58.01 & 337.92 & 144.62 & 1.29 & & 285.37 & +29.41 & 6.19 & $\pm 8^{\prime \prime} .5$ \\
\hline & & $\left(A_{3}\right)$ & $2008 / 06 / 25.71$ & 79.00 & 358.94 & 144.16 & 1.00 & -3.04 & 282.43 & +35.08 & 0.33 & $\pm 8^{\prime \prime} .8$ \\
\hline \multirow[t]{3}{*}{5} & \multirow[t]{3}{*}{ C/2009 L5 } & Cat. & $2009 / 06 / 05.32$ & 75.97 & 355.06 & 144.31 & 1.01 & & 282.16 & +34.47 & 0.90 & 12 \\
\hline & & Grav. & $2010 / 06 / 05.32$ & 75.77 & 354.81 & 144.34 & 1.01 & & 282.14 & +34.41 & 0.96 & $\pm 6^{\prime \prime} .8$ \\
\hline & & $\left(A_{3}\right)$ & $2010 / 06 / 05.33$ & 78.85 & 358.67 & 143.94 & 1.02 & -0.49 & 282.37 & +35.28 & 0.36 & $\pm 6^{\prime \prime} .9$ \\
\hline \multirow[t]{3}{*}{6} & \multirow[t]{3}{*}{ C/2006 J9 } & Cat. & $2006 / 05 / 10.98$ & 86.59 & 9.93 & 143.37 & 1.18 & & 284.18 & +36.55 & 1.75 & 13 \\
\hline & & Grav. & $2006 / 05 / 10.98$ & 86.58 & 9.92 & 143.38 & 1.18 & & 284.18 & $\begin{array}{r}30.00 \\
+36.54\end{array}$ & 1.75 & $\pm 3^{\prime \prime} .9$ \\
\hline & & $\left(A_{3}\right)$ & $2006 / 05 / 10.97$ & 85.59 & 6.94 & 144.00 & 1.06 & +0.83 & 282.39 & +35.88 & 0.76 & $\pm 3^{\prime \prime} .9$ \\
\hline \multirow[t]{3}{*}{7} & \multirow[t]{3}{*}{$\mathrm{C} / 2008 \mathrm{M} 5$} & Cat. & $2008 / 06 / 26.40$ & 99.81 & 22.08 & 139.96 & 0.90 & & 279.35 & +39.34 & 4.97 & 10 \\
\hline & & Grav. & $2008 / 06 / 26.40$ & 99.69 & 21.93 & 140.02 & 0.91 & & 279.37 & +39.30 & 4.92 & $\pm 9^{\prime \prime} .6$ \\
\hline & & $\left(A_{3}\right)$ & $2008 / 06 / 26.37$ & 82.38 & 3.35 & 144.59 & 1.08 & +3.84 & 282.67 & +35.06 & 0.18 & $\pm 9^{\prime \prime} .2$ \\
\hline \multirow[t]{3}{*}{8} & \multirow[t]{3}{*}{$\mathrm{C} / 2008 \mathrm{~K} 8$} & Cat. & $2008 / 05 / 28.65$ & 99.02 & 32.42 & 135.31 & 1.72 & & 289.83 & +43.99 & 10.32 & 14 \\
\hline & & Grav. & $2008 / 05 / 28.65$ & 98.96 & 32.19 & 135.44 & 1.71 & & 289.71 & +43.88 & 10.18 & $\pm 10^{\prime \prime} .2$ \\
\hline & & $\left(A_{3}\right)$ & $2008 / 05 / 28.53$ & 84.47 & 5.59 & 144.45 & 1.01 & +3.25 & 282.38 & +35.36 & 0.38 & $\pm 10^{\prime \prime} .3$ \\
\hline
\end{tabular}

${ }^{\text {a }}$ Cat. =gravitational orbit from catalogue by Marsden \& Williams (2008); Grav. =gravitational orbit computed by us; $\left(A_{3}\right)=$ nongravitational orbit with $A_{3}$ forced to fit the line of reference apsidal orientation as closely as possible.

${ }^{\mathrm{b}}$ Units of $10^{-5} \mathrm{AU}$ day ${ }^{-2}$, a thousand times greater than units used in standard orbital computations; normalized to 1 AU from the Sun.

The iteration proceeded by trial and error until for three chosen values of $A_{3}$, relatively close to one another and preferably equidistant or nearly equidistant, and such that $\left(A_{3}\right)_{1}<\left(A_{3}\right)_{2}<\left(A_{3}\right)_{3}$, the respective offsets, $\epsilon_{1}, \epsilon_{2}$, $\epsilon_{3}$, from the reference apsidal line satisfied a condition $\epsilon_{2}<\min \left(\epsilon_{1}, \epsilon_{3}\right)$. A parabola was then fitted through the three $\epsilon^{2}\left(A_{3}\right)$ points, with the square root of the minimum $\epsilon_{\min }^{2}$ listed in column 12 and the resulting $A_{3}$ value in column 9 of the $\left(A_{3}\right)$ row in Table 4 . The orbital elements, listed in columns $4-8$ of the same row, were then recomputed with this value of $A_{3}$.

Together with Figures 5 and 6 , in which we plot, respectively, the tested sungrazers' inclination and argument of perihelion against their longitude of the ascending node, Table 4 allows us to make a number of conclusions. Most importantly, for every single tested object the introduction of a nongravitational solution leads to a dramatic drop in the offset from the reference apsidal line and the derived angular orbital elements are quite unlike those from the gravitational solution, the magnitude of the differences correlating with the $A_{3}$ parameter. The longitude of the ascending node and the argument of perihelion changed by as much as $\sim 60^{\circ}$ (sic!), the inclination by up to $9^{\circ}$, the perihelion distance by as much as $0.7 R_{\odot}$, and even the perihelion time by up to 0.12 day. The range of orbital differences among the objects was reduced considerably by the nongravitational solutions, from $86^{\circ}$ to $11^{\circ}$ in $\Omega$, from $72^{\circ}$ to $8^{\circ} .5$ in $\omega$, from $9^{\circ}$ to $1^{\circ} .2$ in $i$, and from $0.8 R_{\odot}$ to $0.33 R_{\odot}$ in $q$.
The minimum offset from the reference apsidal line offered by the nongravitational solutions for the eight sungrazers varies from less than $0^{\circ} .2$ to almost $1^{\circ} .4$. Even in the least favorable case is the offset reduced by more than a factor of two compared to the gravitational solution, while more typically the reduction factor is between 10 and 100. And for the seven cases with offsets smaller than $1^{\circ}$, the perihelion distance is confined to a narrow range from $1.00 R_{\odot}$ to $1.08 R_{\odot}$.

Our a priori estimate of $10^{-5} \mathrm{AU}_{\text {day }^{-2}}$ for the parameter $A_{3}$ compares rather favorably with the results. It is within a factor of $\sim 4$ for six of the eight entries in Table 4 and is closer to the lower end of the range. The $A_{3}$ values statistically correlate both with the offsets from the line of apsides and with the longitudes of the ascending node derived from the gravitational solutions. However, as confirmed by our additional tests, there is no functional correlation. The crossover from negative to positive values of $A_{3}$ is near the position of comet C/1843 D1.

Of much interest are the magnitudes of $\left|A_{3}\right|$ at the upper end of their range. The value for comet C/2007 X13 (normalized to a distance of $1 \mathrm{AU}$ from the Sun) is equivalent to $0.503 \mathrm{~cm} \mathrm{~s}^{-2}$, alarmingly close to $0.593 \mathrm{~cm} \mathrm{~s}^{-2}$, the Sun's gravitational acceleration. At a heliocentric distance of about $0.046 \mathrm{AU}$, at which the comet's image was last astrometrically measured, the Sun's gravitational acceleration is $280 \mathrm{~cm} \mathrm{~s}^{-2}$, or $0.140 \mathrm{AU}_{\text {day }}{ }^{-2}$, while the applied nongravitational law predicts for this distance a water-ice sublimation rate 768 times greater 


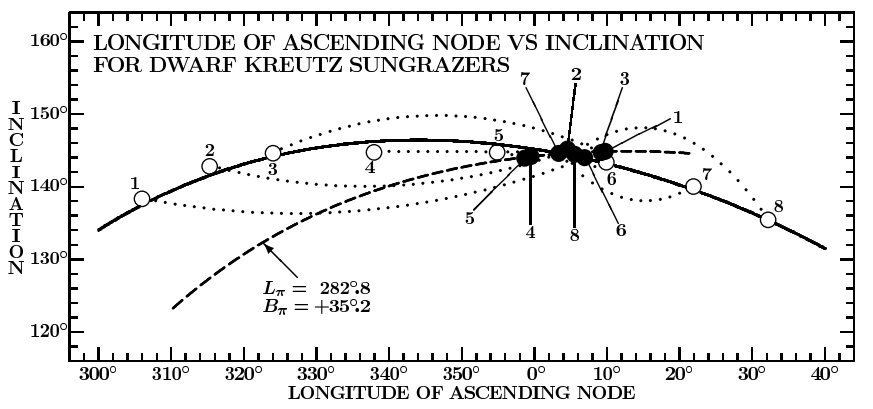

Figure 5. Plot of the longitude of the ascending node against the orbit inclination for eight test dwarf Kreutz sungrazers. The open circles refer to the gravitational solutions (rows Grav. in Table $4)$, the solid circles to the nongravitational solutions in rows $\left(A_{3}\right)$. The number at each circle identifies the sungrazer via column 1 in Table 4. The thick dashed curve is a predicted locus of comets whose orbits fit the reference apsidal line, while the thinner solid curve is its rotated version (curve A in Figure 4). The dotted curves only serve to connect the two types of solution for each object (to show the enormity of the corrections especially in the longitude of the ascending node); their shape is arbitrary.

than at $1 \mathrm{AU}$ from the $\mathrm{Sun}^{5}$ so that the nongravitational acceleration at $0.046 \mathrm{AU}$ was $387 \mathrm{~cm} \mathrm{~s}^{-2}$ or $0.193 \mathrm{AU} \mathrm{day}^{-2}$. The results in Table 4 thus imply that at the end of its visible trajectory, $C / 2007 X 13$ was subjected to a normal component of the nongravitational acceleration that exceeded the Sun's gravitational acceleration by nearly 40 percent! For the other objects in Table 4 the numbers are less extreme but still remarkable.

We should also comment on the relative magnitude of the RMS residuals from the orbital solutions in the last column of Table 4 . Not in a single case is the formal fit from the nongravitational solution markedly worse than from the gravitational solution. Thus, the introduction of $A_{3}$ was tolerable from the data-analysis standpoint; it was of course vital from the standpoint of our dynamical arguments. The equivalence of the gravitational and nongravitational solutions in terms of the RMS residuals is not surprising; it is simply a sign of fairly low accuracy of the astrometric observations. It is the offset from the apsidal line and not the formal quality of fit that is the driver in our undertaking this task and the prime criterion in measuring the significance of the results.

Graphically, the reduction of the ranges of the three angular elements $\Omega, i$, and $\omega$ brought about by the introduction of the nongravitational term with the parameter $A_{3}$, is dramatically revealed by Figures 5 and 6 . From Figure 5 it is obvious that all test sungrazers whose gravitational solutions were distributed along the thickly populated branch associated with $\mathrm{C} / 1843$ D1 are in their nongravitational solutions distributed tightly along the line of apsides instead. All shifts in the test objects' positions in the plot of $\Omega$ against $i$, however large, were accomplished by "sliding" along, not across, the curve.

Our last comment on the results in Table 4 and Figures 5 and 6 is to point out the unanswered questions and employed approximations. Although the nongravitational solutions for the eight test sungrazers appear to

\footnotetext{
5 The nongravitational law predicts that at small heliocentric distances the water-ice sublimation rate (and the sungrazer's momentum-transfer acceleration) varies at a rate slightly steeper than the Sun's gravitational acceleration (Sec. 7).
}

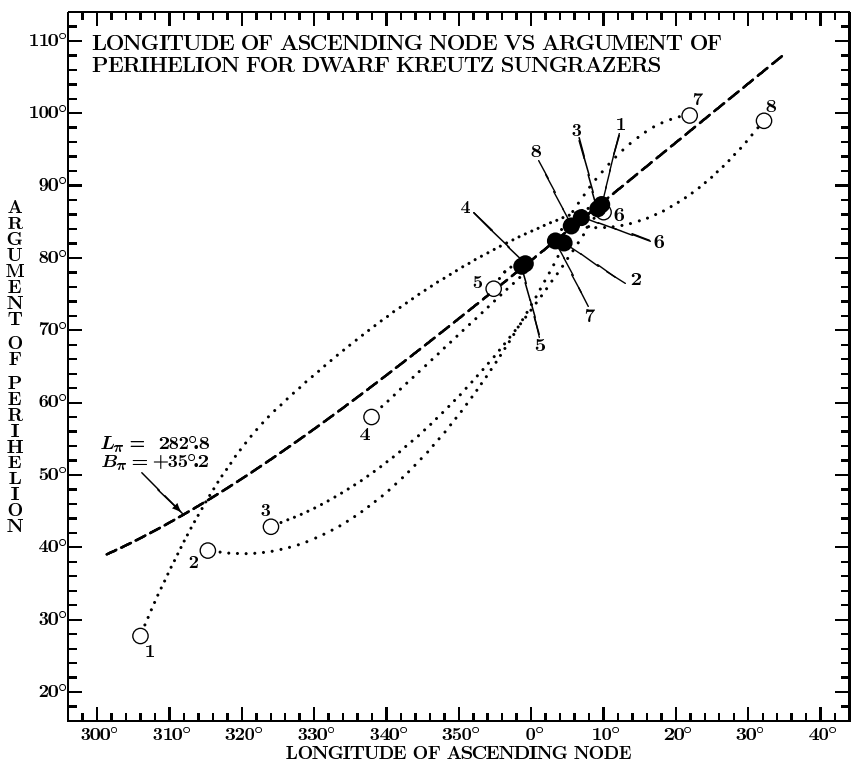

Figure 6. Plot of the longitude of the ascending node against the argument of perihelion for eight test dwarf Kreutz sungrazers. For the description see the caption to Figure 5.

be satisfactory and are responsive to the argument based on the observed trend in $B_{\pi}$ from gravitational solutions, the effects driven by the process of erosion should, in general, include a radial component (parameter $A_{1}$ ) and a transverse component (parameter $A_{2}$ ) as well. While the two components in the orbital plane do not affect the longitude of the ascending node or the inclination directly, the introduction into the equations of motion of their contributions could affect the value of $A_{3}$ as well. We return to this issue, which requires a fuller incorporation of erosion-driven effects, in Sec. 8.

\section{APPARENT SCATTER IN APSIDAL ORIENTATION FROM GRAVITATIONAL ORBITS OF DWARF KREUTZ SUNGRAZERS}

An important question to answer is whether the enormous scatter in the angular orbital elements of the dwarf Kreutz sungrazers derived from the gravitational solutions is only a dynamical effect, as discussed in the previous section or whether there also is a significant contribution from the errors of measurement and/or reduction of the astrometric observations. To address this question, the plot in Figure 7 shows, as a function of a limiting offset $\epsilon_{\text {lim }}$, a cumulative distribution of these objects whose angular offsets $\epsilon$ from the reference apsidal orientation exceed, or are equal to, $\epsilon_{\text {lim }}$.

Figure 7 and Table 5, which summarizes the results from the cumulative distribution, show that the offsets from the reference apsidal orientation are the largest for the set of sungrazers observed with the $\mathrm{C} 2$ coronagraph only. This suggests that the gravitational orbits of the dwarf sungrazers are the most inaccurate when they are based only on astrometric observations from C2 images. This effect could be instrumental, because the orbital arcs in $\mathrm{C} 2$ are shorter and the number of reference stars is sometimes insufficient or their field distribution strongly nonuniform, both of which may lead to inferior orbital solutions. On the other hand, due to the much smaller pixel size of the $\mathrm{C} 2$ coronagraph, the astrometry should 


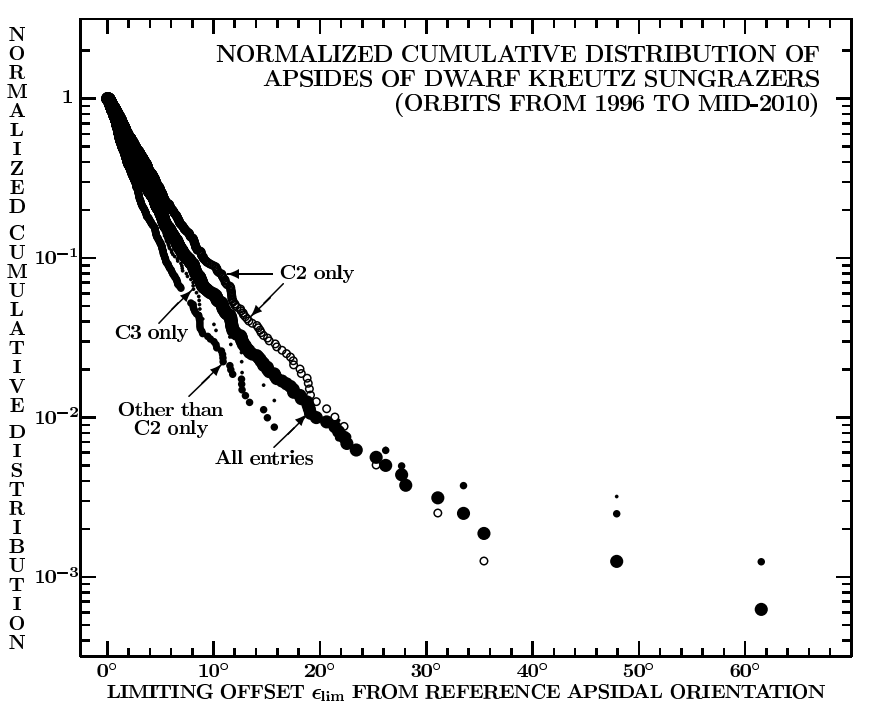

Figure 7. Normalized cumulative distribution of dwarf Kreutz sungrazers with offsets from the reference apsidal orientation equal to or exceeding a limiting offset $\epsilon_{\mathrm{lim}}$. Four sets of the dwarf sungrazers are plotted: (a) a complete set of about 1600 entries (large bullets, marked: All entries); (b) sungrazers with all astrometric positions from images taken only with the $\mathrm{C} 2$ coronagraph on board $S O H O$ (open circles, marked: C2 only); (c) sungrazers with positions from images taken with any $S O H O$ or STEREO coronagraph other than $\mathrm{C} 2$ only (small bullets, marked: Other than C2 only); and (d) sungrazers with all positions from images taken only with the C3 coronagraph on board $\mathrm{SOHO}$ (dots, marked: $\mathrm{C} 3$ only).

be better. Perplexingly, if the greater offsets in Figure 7 were an instrumental effect, one would expect that the C3 only distribution curve should lie below the Other than C2 only distribution, because the latter includes largely the sungrazers whose orbits were based on images from both $\mathrm{C} 3$ and $\mathrm{C} 2$. This expectation is however contradicted in the figure, as the C3 only distribution shows that the orbits based on C3 images yield offsets that are larger than those from the orbits based on C3 plus $\mathrm{C} 2$, so that $\mathrm{C} 2$ images improve the orbits.

We suggest that the solution to this puzzle lies in the same issue that led us to examine a set of representative examples of sungrazers observed only in $\mathrm{C} 2$, that is, at the very end of their trajectory. At this late evolutionary phase, the size of the nucleus of a dwarf sungrazer is near zero because of escalating erosion, and the deviation from a gravitational motion is at a maximum. Gravitational solutions are inappropriate under these circumstances and their failure shows up as an enormous effect in the apsidal orientation.

\section{GENERALIZING THE MOMENTUM-TRANSFER LAW}

Our search for nongravitational orbital solutions in Sec. 5.2 used the Style II formalism of Marsden et al. (1973); the standard nongravitational law, incorporated into this formalism and approximating the sublimation rate of water ice as a function of heliocentric distance, was employed by us deliberately, because this convention allowed us to compare the magnitudes of the nongravitational parameters that we determined for the dwarf Kreutz sungrazers with the magnitudes for ordinary comets at much larger heliocentric distances. This comparison led to our discovery that the ougassing-driven ac-
Table 5

Cumulative Distribution of Apsidal Orientation Offsets of Dwarf Kreutz Sungrazers from Reference Apsidal ORIENTATION (EQ. 2000).

\begin{tabular}{|c|c|c|c|c|}
\hline \multirow{2}{*}{$\begin{array}{l}\text { Offset from } \\
\text { reference apsidal } \\
\text { orientation }^{\mathrm{a}} \\
\epsilon_{\lim }\end{array}$} & \multicolumn{4}{|c|}{$\begin{array}{l}\text { Fraction (\%) of dwarf sungrazers with } \\
\quad \text { apsidal orientation offsets } \epsilon \geq \epsilon_{\text {lim }}\end{array}$} \\
\hline & $\mathrm{All}^{\mathrm{b}}$ & C2 only ${ }^{\mathrm{c}}$ & Other $^{\mathrm{d}}$ & C3 only ${ }^{\mathrm{e}}$ \\
\hline 0.5 & 86 & 94 & 78 & 86 \\
\hline 1.0 & 75 & 83 & 58 & 71 \\
\hline 2.0 & 49 & 60 & 37 & 50 \\
\hline 4.0 & 26 & 35 & 17 & 24 \\
\hline 8.0 & 9 & 27 & 5 & 7 \\
\hline 12.0 & 3.4 & 5.2 & 1.8 & 2.8 \\
\hline Totals & 1598 & 794 & 804 & 313 \\
\hline \multicolumn{5}{|c|}{$\begin{array}{l}\text { a Standard apsidal direction of Kreutz sungrazer system defined by: } \\
\left\langle L_{\pi}\right\rangle=282^{\circ} .8 \text { and }\left\langle B_{\pi}\right\rangle=+35^{\circ} .2 \text {. }\end{array}$} \\
\hline \multicolumn{5}{|c|}{$\begin{array}{l}\mathrm{b} \text { Set of dwarf Kreutz sungrazers detected, measured, and reduced } \\
\text { from images taken with any onboard coronagraph. }\end{array}$} \\
\hline \multirow{2}{*}{\multicolumn{5}{|c|}{$\begin{array}{l}{ }^{\mathrm{c}} \text { Set of dwarf Kreutz sungrazers detected, measured, and reduced } \\
\text { only from images taken with C2 coronagraph on board } S O H O \text {. } \\
\mathrm{d}^{\mathrm{d}} \text { Set of dwarf Kreutz sungrazers detected, measured, and reduced } \\
\text { from images taken with coronagraphs other than only C2. }\end{array}$}} \\
\hline & & & & \\
\hline \multicolumn{5}{|c|}{$\begin{array}{l}\text { 'Set of dwarf Kreutz sungrazers detected, measured, and reduced } \\
\text { only from images taken with C } 3 \text { coronagraph on board SOHO; this } \\
\text { is a subset of the set 'Other'. }\end{array}$} \\
\hline
\end{tabular}

celerations for the dwarf sungrazers were orders of magnitude higher than those for ordinary comets and in the extreme cases comparable in magnitude to the Sun's gravitational acceleration.

The standard momentum-transfer law, $g_{\text {ice }}(r)$, has in Marsden et al.'s (1973) formalism been expressed by an empirical formula,

$$
g_{\text {ice }}(r)=a\left(\frac{r}{r_{0}}\right)^{-m}\left[1+\left(\frac{r}{r_{0}}\right)^{n}\right]^{-k},
$$

where $m=2.15, n=5.093, k=4.6142$, the scaling distance $r_{0}=2.808 \mathrm{AU}$, and the normalization constant $a=0.1113$ such that $g_{\text {ice }}(1 \mathrm{AU})=1$. These constants apply to a so-called isothermal model of water-ice sublimation, which averages the Sun's incident radiation over the surface of a spherical nucleus by assuming that the temperature of the water-ice covered surface does not vary from site to site and depends only on the heliocentric distance. Although the temperature varies over the surface greatly, for the orbit-determination purposes the formula (22) has over the many decades provided excellent service and still is employed nowadays.

Encouraged by the results summarized in Table 4, we continued our experimentation with the nongravitational terms in the equations of motion by testing nongravitational solutions for the dwarf Kreutz sungrazers observed with the $\mathrm{C} 2$ coronagraph by directly incorporating the parameter $A_{3}$, next to the orbital elements, as a variable into the least-squares optimization procedure. Employing Marsden et al.'s (1973) formalism, a few computer runs confirmed our pessimism (Sec. 5.2) that this effort is doomed to failure, given the uncertainties of the $S O H O$ astrometry and very short orbital arcs of the objects observed only with the $\mathrm{C} 2$ coronagraph. We then tested this same approach on extended orbital arcs available for dwarf Kreutz sungrazers observed with both the $\mathrm{C} 2$ and $\mathrm{C} 3$ coronagraphs. For 
a dwarf sungrazer $\mathrm{C} / 2003 \mathrm{Q} 7$, for example, we found $A_{3}=(+0.211 \pm 0.090) \times 10^{-5} \mathrm{AU}_{\mathrm{day}^{-2}}$ - a marginal detection with a $1 \sigma$ relative error of more than 40 percent - by fitting the last 18 of the 45 astrometric positions available. On the other hand, a solution based on all 45 positions led to a completely indeterminate result, $A_{3}=(+0.021 \pm 0.039) \times 10^{-5} \mathrm{AU}_{\mathrm{day}}{ }^{-2}$.

We concluded that to solve for $A_{3}$ as one of the parametric variables directly from the least-squares equations is not the way to proceed. Nevertheless, this experimentation was helpful in that it suggested that (i) the value of $A_{3}$ had a tendency to increase by perhaps as much as one order of magnitude as orbital solutions were derived from astrometric observations closer to the point of the sungrazer's disappearance; (ii) the standard nongravitational law $g_{\text {ice }}(r)$ is not quite appropriate for the dwarf Kreutz sungrazers; and (iii) laws that imply a steeper variation with heliocentric distance are more compatible with the astrometric observations.

Argument (iii) is strongly supported by the fact that numerous species considerably less volatile than water ice, including atomic sodium (e.g., Knight et al. 2010), are known to sublimate profusely at heliocentric distances smaller than $\sim 0.1 \mathrm{AU}$, where dwarf sungrazers are typically observed. It is therefore highly doubtful that the sublimation of water ice typically dominates the erosion process in close proximity to the Sun. Accordingly, the issue of an appropriate momentum-transfer law for the dwarf sungrazers needs comprehensive examination. If the momentum transferred from the outgassing of other, more refractory species should be more important than water ice, the scaling distance should be much smaller than in Eq. $(22), r_{0} \ll 2.8$ AU.

To accommodate a greater number of options and to make the momentum-transfer law more flexible and realistic for applications to dwarf Kreutz sungrazers, it is desirable to fundamentally generalize the momentumtransfer law. In this section we introduce three such laws. One of them, $g_{\mathrm{Na}}(r)$, is based on the sublimation of sodium (derived from the dependence of the saturated vapor pressure on temperature; e.g., Hicks 1963), which is known to outgas profusely from dwarf Kreutz sungrazing comets (e.g., Biesecker et al. 2002; Sekanina 2003; Knight et al. 2010). The resulting sublimation rate as a function of heliocentric distance for the isothermal model can closely be approximated by the formula (22) with the parameters: $m=2.089, n=3.603, k=4.896$, $r_{0}=0.3458 \mathrm{AU}$, and $a=10^{9.145}$ (see also Sekanina \& Kracht 2014). Although the scaling distance $r_{0}$ is much smaller than for water ice, in a range of heliocentric distances where the dwarf Kreutz sungrazers are typically observed, sodium and water ice sublimate at rates that have a similar dependence on $r$. We would therefore expect that the two laws should lead to similar results, a circumstance that is useful in checking the validity of computations.

The second employed momentum-transfer law refers to one of very highly refractory materials - forsterite, the magnesium-rich end-member of the olivine solid solution series $\left(\mathrm{Mg}_{2} \mathrm{SiO}_{4}\right)$, a common silicate in comets, including Kreutz sungrazers (e.g., Sekanina 2000, Kimura et al. 2002, Ciaravella et al. 2010, Sekanina \& Chodas 2012). From the data of Hashimoto's (1990) laboratory experiments, we computed the sublimation rate of forsterite as a function of heliocentric distance in the isothermal case and found that it, too, can closely be approximated by the expression (22) with the parameters: $m=2.634$, $n=5.155, \quad k=3.320, \quad r_{0}=0.014861 \mathrm{AU}=3.1926 R_{\odot}$, and $a=10^{36.10}$ (see also Sekanina \& Kracht 2014). We refer to this law as a $g_{\text {for }}(r)$ law. We find that at a distance as close to the Sun as $5 R_{\odot}, g_{\text {for }}(r)$ varies as steeply as $\sim r^{-18.2}$, that is, very differently from the expected laws for water ice and sodium.

The third law introduced in this section is a generic one, which we refer to as a modified nongravitational law $g_{\text {mod }}\left(r ; r_{0}\right)$ [again normalized to $g_{\bmod }\left(1 \mathrm{AU} ; r_{0}\right)=1$ ] and which is aimed at obtaining the best possible fit by the law of type (22) to the astrometric observations by varying only the scaling distance $r_{0}$. This approach is justified by Marsden et al.'s (1973) finding that the shapes of normalized sublimation curves for a variety of species are fairly similar except for major horizontal shifts in a plot of $\log$ (sublimation rate) against $\log r$, which means that in terms of the approximation formula (22) the curves are relatively insensitive to the exponents $m$ (which always slightly exceeds 2 ), $n$, and $k$, but highly sensitive to the scaling distance $r_{0}$. On some assumptions (see Sekanina \& Kracht 2014), $r_{0}$ measures essentially the heat of sublimation $L$ of the outgassing substance (or a mean value, if more species are involved), varying to a first approximation inversely as the square of $L$,

$$
r_{0} \simeq\left(\frac{\text { const }}{L}\right)^{2},
$$

where a calibration by water ice gives for the constant $19100 \mathrm{AU}^{\frac{1}{2}} \mathrm{cal} \mathrm{mol}^{-1}$ in the case of an isothermal model.

To test the modified nongravitational law on the eight dwarf Kreutz sungrazers, we retain the values of $m, n$, and $k$ for water ice from Eq. (22), but conduct in each case a search for an optimum solution by varying the scaling distance $r_{0}$ until a minimum offset from the reference apsidal orientation is found, as described in Sec. 5.2.

The optimum nongravitational solutions, derived with the four different nongravitational laws, $g_{\text {ice }}(r), g_{\mathrm{Na}}(r)$, $g_{\text {for }}(r)$, and $g_{\bmod }\left(r ; r_{0}\right)$, are compared in Table 6 . The data for the $g_{\text {ice }}(r)$ law are taken over from the $\left(A_{3}\right)$ rows of Table 4, except that the nongravitational parameter in the direction normal to the orbit plane, now denoted $\mathcal{A}_{3}$, is referred to a heliocentric distance of $10 R_{\odot}$ (and expressed in AU day ${ }^{-2}$ ).

Inspection of Table 6 suggests the following: (i) the resulting values of $\mathcal{A}_{3}$ from the solutions based on the standard law and the sodium sublimation law are practically identical, as expected, for all eight test sungrazers; (ii) the nongravitational acceleration for C/2007 X13 at $10 R_{\odot}$ from the Sun exceeds the Sun's gravitational acceleration by up to nearly 40 percent; for the other seven comets it is smaller, but it always amounts to more than 1 percent of the Sun's attraction; (iii) for five comets (C/2007 X13, C/2007 X3, C/2008 M4, C/2006 J9, and $\mathrm{C} / 2008 \mathrm{~K} 8$ ) the modified nongravitational law provides the best solution in terms of the apsidal-line offset; while for $\mathrm{C} / 2001 \mathrm{Y} 4$ the solutions based on the modified law, the standard law, and the sodium sublimation law all fit the data equally well; no minimum apsidal-line offset was found among the solutions based on the modified law for C/2009 L5 and C/2008 M5; (iv) for C/2009 L5 the 
Nongravitational Motions of Dwarf Kreutz Sungrazers

Table 6

Comparison of the Momentum-Transfer Laws $g_{\text {ice }}(r), g_{\mathrm{Na}}(r), g_{\mathrm{for}}(r)$, and $g_{\mathrm{mod}}\left(r ; r_{0}\right)$ in Fitting the Orbits of the Eight Dwarf Sungrazers from Table 4.

\begin{tabular}{|c|c|c|c|c|c|c|c|c|c|}
\hline \multirow[b]{2}{*}{ No. } & \multirow[b]{2}{*}{ Object } & \multirow{2}{*}{$\begin{array}{l}\text { Momentum } \\
\text { transfer } \\
\text { law }\end{array}$} & \multirow{2}{*}{$\begin{array}{l}\text { Param- } \\
\text { eter } \mathcal{A}_{3} \\
\left(\text { units }^{\mathrm{a}}\right) \\
\end{array}$} & \multicolumn{2}{|c|}{ Distance $r_{0}{ }^{\mathrm{b}}$} & \multicolumn{2}{|c|}{ Line of apsides } & \multirow{2}{*}{$\begin{array}{l}\text { Apsidal } \\
\text { line's } \\
\text { offset } \\
\end{array}$} & \multirow{2}{*}{$\begin{array}{c}\text { RMS } \\
\text { resid- } \\
\text { ual } \\
\end{array}$} \\
\hline & & & & in $\mathrm{AU}$ & in $R_{\odot}$ & $L_{\pi}$ & $B_{\pi}$ & & \\
\hline 1 & C/2007 X13 & $\begin{array}{l}g_{\text {ice }}(r) \\
g_{\mathrm{Na}}(r) \\
g_{\text {for }}(r) \\
g_{\text {mod }}\left(r ; r_{0}\right)\end{array}$ & $\begin{array}{l}-0.188 \\
-0.189 \\
-0.151 \\
-0.183\end{array}$ & $\begin{array}{l}\ldots \ldots \\
\ldots \ldots \\
\ldots \ldots \\
0.126\end{array}$ & $\begin{array}{l}\ldots \ldots \\
\ldots \ldots \\
\ldots \ldots \\
27.1\end{array}$ & $\begin{array}{l}282^{\circ} .95 \\
282.99 \\
281.88 \\
282.81\end{array}$ & $\begin{array}{l}+35^{\circ} .08 \\
+35.08 \\
+35.20 \\
+35.20\end{array}$ & $\begin{array}{l}0^{\circ} .17 \\
0.20 \\
0.75 \\
0.01\end{array}$ & $\begin{array}{l} \pm 13^{\prime \prime} .0 \\
\pm 13.1 \\
\pm 13.2 \\
\pm 12.9\end{array}$ \\
\hline 2 & C/2007 X3 & $\begin{array}{l}g_{\text {ice }}(r) \\
g_{\mathrm{Na}}(r) \\
g_{\text {for }}(r) \\
g_{\text {mod }}\left(r ; r_{0}\right)\end{array}$ & $\begin{array}{l}-0.111 \\
-0.111 \\
-0.0254 \\
-0.0121\end{array}$ & $\begin{array}{c}\ldots \ldots \\
\ldots \ldots \\
\ldots \ldots \\
0.0056\end{array}$ & $\begin{array}{c}\ldots \ldots \\
\ldots \ldots \\
\ldots \ldots \\
1.2\end{array}$ & $\begin{array}{l}284.17 \\
284.18 \\
283.13 \\
283.08\end{array}$ & $\begin{array}{l}+34.44 \\
+34.42 \\
+35.13 \\
+35.15\end{array}$ & $\begin{array}{l}1.36 \\
1.37 \\
0.28 \\
0.23\end{array}$ & $\begin{array}{l} \pm 6.6 \\
\pm 6.6 \\
\pm 3.0 \\
\pm 2.8\end{array}$ \\
\hline 3 & C/2001 Y4 & $\begin{array}{l}g_{\text {ice }}(r) \\
g_{\mathrm{Na}}(r) \\
g_{\text {for }}(r) \\
g_{\text {mod }}\left(r ; r_{0}\right)\end{array}$ & $\begin{array}{l}-0.0315 \\
-0.0312 \\
-0.0186 \\
-0.0313\end{array}$ & $\begin{array}{l}\ldots \ldots \\
\ldots \ldots \\
\ldots \ldots \\
2.12\end{array}$ & $\begin{array}{c}\ldots \ldots \\
\ldots \ldots \\
\ldots \ldots \\
455\end{array}$ & $\begin{array}{l}283.15 \\
283.15 \\
283.73 \\
283.15\end{array}$ & $\begin{array}{l}+35.26 \\
+35.24 \\
+35.21 \\
+35.23\end{array}$ & $\begin{array}{l}0.29 \\
0.29 \\
0.76 \\
0.29\end{array}$ & $\begin{array}{l} \pm 20.8 \\
\pm 20.8 \\
\pm 21.0 \\
\pm 20.8\end{array}$ \\
\hline 4 & C/2008 M4 & $\begin{array}{l}g_{\text {ice }}(r) \\
g_{\mathrm{Na}}(r) \\
g_{\text {for }}(r) \\
g_{\text {mod }}\left(r ; r_{0}\right)\end{array}$ & $\begin{array}{l}-0.0228 \\
-0.0227 \\
-0.1004 \\
-0.1388\end{array}$ & $\begin{array}{l}\ldots \ldots \\
\ldots \ldots \\
\ldots \ldots \\
0.012\end{array}$ & $\begin{array}{c}\ldots \ldots \\
\ldots \ldots \\
\ldots \ldots \\
2.6\end{array}$ & $\begin{array}{l}282.43 \\
282.42 \\
282.63 \\
282.65\end{array}$ & $\begin{array}{l}+35.08 \\
+35.10 \\
+35.15 \\
+35.15\end{array}$ & $\begin{array}{l}0.33 \\
0.33 \\
0.15 \\
0.13\end{array}$ & $\begin{array}{l} \pm 8.8 \\
\pm 8.8 \\
\pm 8.5 \\
\pm 8.4\end{array}$ \\
\hline 5 & C/2009 L5 ${ }^{\mathrm{c}}$ & $\begin{array}{l}g_{\text {ice }}(r) \\
g_{\mathrm{Na}}(r) \\
g_{\text {for }}(r)\end{array}$ & $\begin{array}{l}-0.0037 \\
-0.0036 \\
-0.0046\end{array}$ & $\begin{array}{l}\ldots \ldots \\
\ldots \ldots \\
\ldots \ldots\end{array}$ & & $\begin{array}{l}282.37 \\
282.37 \\
282.37\end{array}$ & $\begin{array}{l}+35.28 \\
+35.28 \\
+35.29\end{array}$ & $\begin{array}{l}0.36 \\
0.36 \\
0.36\end{array}$ & $\begin{array}{l} \pm 6.9 \\
\pm 6.9 \\
\pm 6.6\end{array}$ \\
\hline 6 & C/2006 J9 & $\begin{array}{l}g_{\text {ice }}(r) \\
g_{\mathrm{Na}}(r) \\
g_{\text {for }}(r) \\
g_{\text {mod }}\left(r ; r_{0}\right)\end{array}$ & $\begin{array}{l}+0.0062 \\
+0.0062 \\
+0.0024 \\
+0.0067\end{array}$ & $\begin{array}{l}\ldots \ldots \\
\ldots \ldots \\
\ldots \ldots \\
0.052\end{array}$ & $\begin{array}{l}\ldots \ldots \\
\ldots \ldots \\
\ldots \ldots \\
11.2\end{array}$ & $\begin{array}{l}282.39 \\
282.39 \\
283.48 \\
282.44\end{array}$ & $\begin{array}{l}+35.88 \\
+35.88 \\
+36.24 \\
+35.78\end{array}$ & $\begin{array}{l}0.76 \\
0.76 \\
1.18 \\
0.65\end{array}$ & $\begin{array}{l} \pm 3.9 \\
\pm 3.9 \\
\pm 3.9 \\
\pm 3.9\end{array}$ \\
\hline 7 & $\mathrm{C} / 2008 \mathrm{M} 5^{\mathrm{d}}$ & $\begin{array}{l}g_{\text {ice }}(r) \\
g_{\mathrm{Na}}(r) \\
g_{\text {for }}(r)\end{array}$ & $\begin{array}{l}+0.0288 \\
+0.0287 \\
+0.0745\end{array}$ & $\begin{array}{l}\ldots \ldots \\
\ldots \ldots \\
\ldots \ldots\end{array}$ & $\begin{array}{l}\ldots \ldots \\
\ldots \ldots \\
\ldots \ldots\end{array}$ & $\begin{array}{l}282.67 \\
282.67 \\
281.96\end{array}$ & $\begin{array}{l}+35.06 \\
+35.06 \\
+35.05\end{array}$ & $\begin{array}{l}0.18 \\
0.17 \\
0.70\end{array}$ & $\begin{array}{l} \pm 9.2 \\
\pm 9.2 \\
\pm 9.5\end{array}$ \\
\hline 8 & $\mathrm{C} / 2008 \mathrm{~K} 8^{\mathrm{e}}$ & $\begin{array}{l}g_{\text {ice }}(r) \\
g_{\mathrm{Na}}(r) \\
g_{\text {mod }}\left(r ; r_{0}\right)\end{array}$ & $\begin{array}{l}+0.0243 \\
+0.0242 \\
+0.0284\end{array}$ & $\begin{array}{l}\ldots \ldots \\
\ldots \ldots \\
0.080\end{array}$ & $\begin{array}{l}\ldots \ldots \\
\ldots \ldots \\
17.2\end{array}$ & $\begin{array}{l}282.38 \\
282.39 \\
282.49\end{array}$ & $\begin{array}{l}+35.36 \\
+35.38 \\
+35.33\end{array}$ & $\begin{array}{l}0.38 \\
0.38 \\
0.29\end{array}$ & $\begin{array}{l} \pm 10.3 \\
\pm 10.3 \\
\pm 10.4\end{array}$ \\
\hline
\end{tabular}

a Normal component of the momentum-transfer acceleration at a heliocentric distance of $10 R_{\odot}$, expressed in $\mathrm{AU}^{-2}$ day $^{-2}$. The Sun's gravitational acceleration at this distance is $0.1366 \mathrm{AU}^{\text {day }}{ }^{-2}$.

b By definition, scaling distance $r_{0}$ is always equal to $2.808 \mathrm{AU}=603 R_{\odot}$ for law $g_{\text {ice }}(r) ; 0.3458 \mathrm{AU}=74.3 R_{\odot}$ for $g_{\mathrm{Na}}(r)$; and $0.01486 \mathrm{AU}=3.19 R_{\odot}$ for $g_{\text {for }}(r)$ [Sekanina \& Kracht 2014]

$\mathrm{c}$ There was no minimum offset from the reference apsidal line among modified solutions with $r_{0}$ between 0.02 and 4 AU,

There was no minimum offset from the reference apsidal line among modified solutions with $r_{0}$ between 0.02 and 4 AU,
although the RMS residual was decreasing steadily with decreasing $r_{0}$, which is consistent with the lower RMS residual from the solution with the forsterite-based sublimation law.

$\mathrm{d}$ There was no minimum offset from the reference apsidal line among modified solutions with $r_{0}$ between 0.02 and 2.8 AU.

e The solution optimized for this object with law $g_{\text {for }}(r)$ resulted in $A_{3}=0$, i.e., in a gravitational solution (Table 4 ).

ice, sodium, and forsterite sublimation laws offer equally good solutions in terms of the apsidal-line offset, but the solution based on the forsterite sublimation law provides a fit with a RMS residual superior to those from the solutions based on the other two laws; (v) for C/2008 M5, the best fit results by a narrow margin from the solution based on the sodium sublimation law; (vi) the forsterite sublimation law does not work for $\mathrm{C} / 2008 \mathrm{~K} 8$; (vii) in terms of the scaling distance of the modified law, the comets are divided into three groups: two (C/2007 X3 and $\mathrm{C} / 2008 \mathrm{M} 4$ ) have the nongravitational variations steeper than even the forsterite sublimation law; three (C/2006 J9, C/2008 K8, and C/2007 X13) have the variations steeper than the sodium sublimation law, but less steep than the forsterite sublimation law; and only one (C/2001 Y4) has variations less steep than the sodium law, though still steeper than the ice sublimation law.

To summarize, in terms of the apsidal-line offset and the RMS residual, the water-ice sublimation law proves competitive with the other three laws only in the case of
$\mathrm{C} / 2001$ Y4. For the remaining seven comets, the laws with smaller scaling distances, that is, implying steeper variations, are superior, suggesting that momentumtransfer effects driven by the sublimation of species substantially less volatile than water ice dominate the motions of these Kreutz sungrazers.

\section{THREE-PARAMETER NONGRAVITATIONAL SOLUTIONS FOR DWARF KREUTZ SUNGRAZERS}

In Sec. 5.2 we already remarked that among comets with perihelion distances of $\gtrsim 1$ AU that required an incorporation of the nongravitational terms into the equations of motion, almost never was there the need to include a normal component, $A_{3}$. One of very few exceptions was the case of comet $71 \mathrm{P} /$ Clark, for which inclusion of $A_{3}$ was necessary in order to link the apparitions 1995-2000 and 1995-2006 (Nakano 2001, 2006, 2008).

From what we have until now determined in this paper, the dwarf sungrazers of the Kreutz system are a major exception to the rule of $A_{3} \rightarrow 0$ : the normal component 
always appears to play a role in their orbital motions. However, since we have not up to this point investigated the contributions from $A_{1}$ and $A_{2}$ (both of which having been assumed zero), it is unclear whether or not the normal component actually dominates the other two in magnitude.

Since the nongravitational parameters $A_{1}, A_{2}$, and $A_{3}$ cannot satisfactorily be determined in the course of optimizing an orbital solution by directly incoporating them as parametric variables into the equations of motion when fitting the astrometric observations, it is necessary to explore this issue by employing iterations.

We begin with the relations (3) between the instantaneous rates of change in the angular orbital elements, $d \omega / d t, d \Omega / d t$, and $d i / d t$ on the one hand and the acceleration components, which we now express as a function of a dimensionless, normalized nongravitational law $g(r)$, on the other hand. We note that $g(r)$ stands for any of the $g_{\text {ice }}(r), \ldots, g_{\bmod }\left(r ; r_{0}\right)$ laws:

$$
\left(\begin{array}{c}
j_{\mathrm{R}}(t) \\
j_{\mathrm{T}}(t) \\
j_{\mathrm{N}}(t)
\end{array}\right)=\left(\begin{array}{l}
A_{1} \\
A_{2} \\
A_{3}
\end{array}\right) \cdot g(r)
$$

Integrating over the period of observation, from $t_{\mathrm{beg}}$ to $t_{\text {fin }}$, we find for the overall increments in the three angular elements:

$$
\left(\begin{array}{c}
\Delta \omega \\
\Delta \Omega \\
\Delta i
\end{array}\right)=\left(\begin{array}{ccc}
\Im_{11} & \Im_{21} & \Im_{31} \\
0 & 0 & \Im_{32} \\
0 & 0 & \Im_{33}
\end{array}\right) \cdot\left(\begin{array}{l}
A_{1} \\
A_{2} \\
A_{3}
\end{array}\right)
$$

where

$$
\begin{aligned}
& \Im_{11}=\int_{t_{\mathrm{beg}}}^{t_{\mathrm{fin}}} X_{\mathrm{R}} g(r) d t, \\
& \Im_{21}=\int_{t_{\mathrm{beg}}}^{t_{\mathrm{fin}}} X_{\mathrm{T}} g(r) d t, \\
& \Im_{31}=\int_{t_{\mathrm{beg}}}^{t_{\mathrm{fin}}} X_{\mathrm{N}} g(r) d t, \\
& \Im_{32}=\int_{t_{\mathrm{beg}}}^{t_{\mathrm{fin}}} Y_{\mathrm{N}} g(r) d t, \\
& \Im_{33}=\int_{t_{\mathrm{beg}}}^{t_{\mathrm{fin}}} Z_{\mathrm{N}} g(r) d t,
\end{aligned}
$$

and $r=r(t)$. Inserting Eqs. (25) and (26) into the first of Eqs. (9), the established constraint $\Delta L_{\pi}=0$ offers the following relationship among $A_{1}, A_{2}$, and $A_{3}$ :

$$
A_{1} \Im_{11}+A_{2} \Im_{21}+A_{3} \Im_{31}^{*}=0
$$

where

$$
\Im_{31}^{*}=\Im_{31}+\Im_{32} \frac{1-\sin ^{2} \omega \sin ^{2} i}{\cos i}-\frac{1}{2} \Im_{33} \sin 2 \omega \tan i .
$$

In reality, the constraint $\Delta L_{\pi}=0$ is of course valid only statistically, as Figure 3 and Tables 2 and 3 show. Since $A_{3} \neq 0$ and since the type of solutions investigated up to now have been based on a constraint $A_{1}=A_{2}=0$, condition (27) inevitably requires that $\Im_{31}^{*} \simeq 0$, in which case

$$
A_{1} \Im_{11}+A_{2} \Im_{21} \simeq 0
$$

This relation shows that there is no reason why $A_{1}$ and $A_{2}$ should be zero; in fact, there is an infinite number of nonzero $\left(A_{1}, A_{2}\right)$ pairs that satisfy the condition (29). Some of the pairs, in which $A_{1} \neq 0$ and $A_{2} \neq 0$, may provide even a better match to the reference apsidal line orientation than does the case $A_{1}=A_{2}=0$. To determine what pair of $A_{1}$ and $A_{2}$ offers - on the statistically valid condition of $\Delta L_{\pi}=0$ - a solution optimized in terms of a minimum apsidal offset is the final objective of this investigation.

The contributions to the orbital solution from the parameter $A_{3}$ on the one hand and from $A_{1}$ and $A_{2}$ on the other hand are now separated from each other, and the extension of our work - a transition from solutions with $A_{3}$ to those with all three parameters - is accomplished with the aid of Eq. (29). Keeping $A_{3}$ constant and equivalent to $\mathcal{A}_{3}$ in Table 6 , we continue to search for a pair of $A_{1}$ and $A_{2}$ such that it results in a minimum apsidal-line offset; we successively iterate $A_{1}$ and find $A_{2}$ from

$$
A_{2} \simeq-\frac{\Im_{11}}{\Im_{21}} A_{1}
$$

with $\Im_{11}$ and $\Im_{21}$ computed from Eqs. (26) by numerical integration of the expressions, in which the $g(r)$ again stands for any of the employed nongravitational laws. Once a minimum offset from the apsidal line is found for an adopted $A_{3}$, the resulting $A_{1}$ and $A_{2}$ are kept constant and a search for a new $A_{3}$ initiated by further optimizing the apsidal line, etc., until the offset's ultimate minimum is found. In practice, the momentum-transfer law selected for this approach should be the one providing the least offset from the reference apsidal orientation in Table 6, which, as it turns out, is in most cases the modified law $g_{\text {mod }}$.

The results of these computations are presented in Table 7 , which shows that our final sets of orbital elements for all eight test sungrazers match the reference direction of the line of apsides to within $0^{\circ} .2$, which is - as seen from Table 1 its intrinsic uncertainty. For two comets in Table 7, C/2007 X13 and C/2008 M5, no threeparameter nongravitational solution has been attempted, because a single-parameter solution already implies an offset smaller than the stipulated limit of $0^{\circ} .2$. For the remaining entries of Table 7 , the three-parameter solution was successfully carried out, yielding in most cases the radial component positive and dominating the other two components. The exceptions are $\mathrm{C} / 2007 \mathrm{X} 3$ and $\mathrm{C} / 2008 \mathrm{M} 4$, for which the radial component came out to be negative, for which we do not have an explanation.

The sungrazers C/2007 X3 and C/2008 M4 are, together with $\mathrm{C} / 2009$ L5, the objects in Tables 6 and 7 with the most steeply varying nongravitational accelerations, characterized by the smallest scaling distances $r_{0}$. Next comes another group of three - C/2006 J9, C/2007 X13, and $\mathrm{C} / 2008 \mathrm{~K} 8$ - whose scaling distances are in a range from $0.05 \mathrm{AU}$ to $0.15 \mathrm{AU}$, intermediate between those of the forsterite sublimation law and the sodium sublimation law. The equivalent sublimation heat is estimated at 50000 to $80,000 \mathrm{cal} \mathrm{mol}^{-1}$. The motion of C/2008 M5 was fitted best by the sodium sublimation law, and the volatile end is represented by $\mathrm{C} / 2001 \mathrm{Y} 4$, whose motion appears to have been affected by sublimation of species that may have included water ice; the effective sublima- 
Table 7

Final Nongravitational Orbital Elements for Eight Test Dwarf Kreutz Sungrazers (Condition $\Delta L_{\pi}=0$; Equinox J2000).

\begin{tabular}{|c|c|c|c|c|c|c|c|c|c|c|c|c|c|c|c|}
\hline \multirow[b]{2}{*}{ No. } & \multirow[b]{2}{*}{ Object } & \multicolumn{5}{|c|}{ Orbital elements ${ }^{\mathrm{a}}$} & \multicolumn{5}{|c|}{ Nongravitational law and parameters ${ }^{\mathrm{b}}$} & \multicolumn{2}{|c|}{ Line of apsides } & \multirow{2}{*}{$\begin{array}{c}\text { Apsidal } \\
\text { line's } \\
\text { offset }\end{array}$} & \multirow{2}{*}{$\begin{array}{l}\text { RMS } \\
\text { resid- } \\
\text { ual }\end{array}$} \\
\hline & & $t_{\pi}(\mathrm{ET})$ & $\omega$ & $\Omega$ & $i$ & $q$ & law & {$\left[r_{0}\right]^{\mathrm{c}}$} & $\mathcal{A}_{1}$ & $\mathcal{A}_{2}$ & $\mathcal{A}_{3}$ & $L_{\pi}$ & $B_{\pi}$ & & \\
\hline 1 & /2007 X13 & $2007 / 12 / 14.523$ & 8 & $9^{\circ} .56$ & $44^{\circ} .753$ & 1.023 & MD & 0.12 & & & & $282^{\circ} .81$ & & 0.01 & $\pm 12^{\prime \prime}$. \\
\hline 2 & /2007 X3 & 219 & 8 & & 4.210 & 1.211 & MD & 0.0056 & $(-0.0$ & +0 . & & & & 0.00 & \pm 2.3 \\
\hline 3 & /2001 Y4 & 662 & 83.41 & 4.961 & 144.505 & 1.020 & $\mathrm{MD}$ & & +0.0 & -1 & & 283.04 & .23 & 0.19 & \pm 21.8 \\
\hline 4 & C/2008 M4 & 2008 & 78.839 & 359.0 & 144.0 & 1.013 & $\mathrm{MD}$ & 0.012 & $(-0$. & +0 . & & 32.80 & & 0.00 & \pm 8.3 \\
\hline 5 & C/2009 L5 & 2009 & 78.5 & 358.534 & 143.8 & 1.031 & $\mathrm{FT}$ & & & -0 & & & & 0.18 & \pm 6.2 \\
\hline 6 & C/2006 J9 & $2006 /$ & 83.8 & & 144. & 1.058 & MD & 0.052 & +0.0246 & -0.0044 & & 68 & & 0.17 & \pm 4.1 \\
\hline 7 & C/2008 M5 & & & & & 1.082 & NA & & & & & & & 0.17 & \pm 9.2 \\
\hline 8 & C/2008 K8 & $2008 / 05 / 28.534$ & 83.630 & 4.996 & 144.562 & 1.032 & MD & 0.080 & +0.0376 & -0.0060 & +0.0110 & 282.80 & +35.19 & 0.01 & \pm 10.2 \\
\hline
\end{tabular}

a Perihelion distance $q$ is expressed in units of solar radii, $R_{\odot}\left(1 R_{\odot}=0.0046548 \mathrm{AU}\right)$.

b $\mathrm{MD}=$ modified law; FT $=$ forsterite sublimation law $\left(r_{0}=0.01486 \mathrm{AU}\right) ; \mathrm{NA}=$ sodium sublimation law $\left(r_{0}=0.3458 \mathrm{AU}\right)$; scaling distance of fitted modified law $r_{0}$ is expressed in $\mathrm{AU}$; the acceleration components $\mathcal{A}_{1}, \mathcal{A}_{2}$, and $\mathcal{A}_{3}$ are referred to heliocentric distance of $10 R_{\odot}$ and are

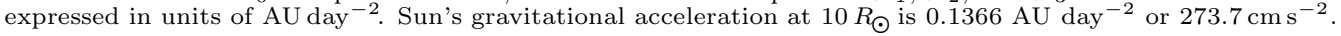

${ }^{\mathrm{c}}$ Distance $r_{0}$ is in $\mathrm{AU}$; in units of $R_{\odot}$, the values are, from top to bottom: 27.1, 1.2, 455, 2.6, 11.2, and 17.2. The range of heliocentric distances spanned by the observations with the C2 coronagraph is: $0.0587-0.0461 \mathrm{AU}$ (or $12.6-9.9 R_{\odot}$ ) for $\mathrm{C} / 2007 \mathrm{X} 13 ; 0.0551-0.0375 \mathrm{AU}$ (or $11.8-8.1 R_{\odot}$ ) for $\mathrm{C} / 2007 \mathrm{X} 3 ; 0.0569-0.0420 \mathrm{AU}$ (or $12.2-9.0 R_{\odot}$ ) for $\mathrm{C} / 2001 \mathrm{Y} 4 ; 0.0581-0.0441 \mathrm{AU}$ (or $12.5-9.5 R_{\odot}$ ) for C/2008 M4; $0.0588-0.0385 \mathrm{AU}$

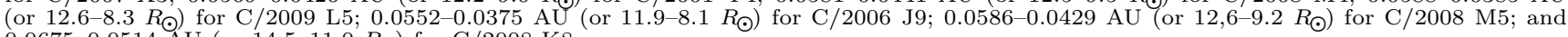
0.0675-0.0514 AU (or 14.5-11.0 $R_{\odot}$ ) for C/2008 K8.

tion heat is estimated at between 13000 and 15000 cal $\mathrm{mol}^{-1}$. Because the laws applicable to the last two sungrazers vary essentially as an inverse square of heliocentric distance along the observed arcs of the orbits, there could be contributions from the solar radiation pressure (but only in $\mathcal{A}_{1}$ of course), if the nuclei of these comets were already shattered into dust at the time.

The very high nongravitational accelerations, already mentioned in Sec. 5.2, are fully confirmed, including the record value of $\mathcal{A}_{3}$ for $\mathrm{C} / 2007 \mathrm{X} 13$, which is 134 percent of the Sun's gravitational acceleration. Similarly, the overall nongravitational accelerations of C/2001 Y4 and C/2009 L5 amount to, respectively, 67 and 51 percent of the Sun's attraction. Even the least overall nongravitational accelerations in Table 7 are still on the order of $\sim 20$ percent of the Sun's attraction.

There are at least three physical processes that operate on the dwarf sungrazers in the final phase of their disintegration near the end of the visible trajectory and are responsible for the very high nongravitational accelerations applied to the test objects in Table 7: sublimation, fragmentation, and the Sun's radiation pressure. The joint contribution to the acceleration from the first two processes, which result in erosion of the sungrazer's nucleus, with a progressive loss rate of its mass, can numerically be simulated by the law $g(r)$, whereas the radiation pressure acceleration varies of course as $r^{-2}$ except at close proximity to the Sun, where it increases as $2\left[1-\sqrt{1-\left(R_{\odot} / r\right)^{2}}\right]$; this rate of variation is 1.03 times steeper than the inverse square law $3 R_{\odot}$ from the Sun, 1.15 times steeper at $1.5 R_{\odot}, 1.29$ times at $1.2 R_{\odot}$, and 1.53 times steeper at $1.05 R_{\odot}$.

We begin with the conservation of momentum law, which requires that a relative mass erosion rate, $\dot{\mathcal{M}}$, of a comet generates an acceleration $\gamma$ on its nucleus of mass $\mathcal{M}$, which at time $t$ satisfies a relation

$$
\gamma(t) \mathcal{M}(t)=-v(t) \kappa(t) \dot{\mathcal{M}}(t),
$$

where $v(t)$ is the outflow velocity of the eroded mass, $\kappa(t)$ is a factor that accounts for the degree of its collimation, and the minus sign indicates that $v(t)$ and $\gamma(t)$, both taken here as positive quantities, point in opposite directions. The acceleration is in the following written in terms of its magnitude at $r_{*}=r\left(t_{*}\right)=10 R_{\odot}$,

$$
\gamma(t)=\mathcal{A}_{*} \frac{g(r)}{g\left(r_{*}\right)},
$$

where $r=r(t)$ and $\mathcal{A}_{*}=\mathcal{A}\left(r_{*}\right)=\sqrt{\mathcal{A}_{1}^{2}+\mathcal{A}_{2}^{2}+\mathcal{A}_{3}^{2}}$ from columns 10 to 12 of Table 7 , while the nongravitational law, from column 8 , is at the heliocentric distances $r$ and $r_{*}$ equal to, respectively, $g(r)$ and $g\left(r_{*}\right)$. Because the observed orbital arcs of the test sungrazers are very short, we conveniently approximate the nongravitational law by a "local" power law,

$$
\gamma(t)=\mathcal{A}_{*}\left(\frac{r_{*}}{r}\right)^{\zeta}
$$

where an effective exponent $\zeta(r)$ near $r(t)$ is related to the parameters of the $g(r)$-type law from Eq. (22) by

$$
\zeta(r)=m+\frac{n k}{1+\left(r_{0} / r\right)^{n}} .
$$

Next, we approximate $v(t)$ by a thermal velocity, which equals to $v_{\mathrm{th}}(r)=v_{0}(1 \mathrm{AU} / r)^{\frac{1}{4}}$. At $1 \mathrm{AU}$ from the Sun, we find $v_{0}$ equal to $0.44 \mathrm{~km} \mathrm{~s}^{-1}$ for forsterite, $0.78 \mathrm{~km} \mathrm{~s}^{-1}$ for sodium atoms, and $0.54 \mathrm{~km} \mathrm{~s}^{-1}$ for water ice. Averaging, we accept $\bar{v}_{0} \approx 0.6 \mathrm{~km} \mathrm{~s}^{-1}$.

The range of possible values for the collimation factor is $0 \leq \kappa \leq 1$. Having no clue for preferring any particular value, we adopt, conditionally, $\bar{\kappa} \approx 0.5$.

With the help of Eq. (33) and these approximations, we now write Eq. (31) after integration over an interval of observations, from $t_{\mathrm{beg}}$ to $t_{\mathrm{fin}}$,

$$
\log _{\mathrm{e}} \frac{\mathcal{M}\left(t_{\mathrm{fin}}\right)}{\mathcal{M}\left(t_{\mathrm{beg}}\right)}=-\frac{\mathcal{A}_{*} r_{*}^{\zeta}}{\bar{v}_{0} \bar{\kappa}}(1 \mathrm{AU})^{-\frac{1}{4}} \int_{t_{\mathrm{beg}}}^{t_{\mathrm{fin}}} r^{\frac{1}{4}-\zeta} d t .
$$

The integral on the right-hand side can be solved by replacing time $t$ with a dimensionless variable, involving the perihelion distance $q$ and a heliocentric distance $r(t)$,

$$
x(t)=\frac{q}{r(t)} .
$$


An interval between a preperihelion time $t$ and the perihelion time $t_{\pi}$ is for a parabolic motion related to $x$ by

$$
t-t_{\pi}=-c_{0} q^{\frac{3}{2}} x^{-\frac{3}{2}}(1+2 x)(1-x)^{\frac{1}{2}},
$$

with $c_{0}=27.38$ day $\mathrm{AU}^{-\frac{3}{2}}$. Differentiating Eq. (37) and inserting $d x$ for $d t$ in Eq. (35), we find

$$
\log _{\mathrm{e}} \frac{\mathcal{M}\left(t_{\mathrm{fin}}\right)}{\mathcal{M}\left(t_{\mathrm{beg}}\right)}=-\frac{c_{1} q^{\frac{7}{4}} \mathcal{A}_{*}}{\bar{v}_{0} \bar{\kappa}}\left(\frac{r_{*}}{q}\right)^{\zeta} \int_{x_{\mathrm{beg}}}^{x_{\mathrm{fin}}} x^{\zeta-\frac{11}{4}}(1-x)^{-\frac{1}{2}} d x
$$

where $c_{1}=\frac{3}{2} c_{0}(1 \mathrm{AU})^{-\frac{1}{4}}=41.07$ day $\mathrm{AU}^{-\frac{7}{4}}, \bar{v}_{0}$ is in $\mathrm{AU}$ day $^{-1}$, and $x_{\text {beg }}$ and $x_{\text {fin }}$ satisfy Eq. (36). Writing the integral in terms of the incomplete beta function and expressing $\bar{v}_{0}$ in $\mathrm{km} \mathrm{s}^{-1}$, the solution becomes

$$
\frac{\mathcal{M}_{\mathrm{fin}}}{\mathcal{M}_{\mathrm{beg}}}=\exp \left\{\frac{C \mathcal{A}_{*}^{r_{*}^{\zeta}}}{\bar{v}_{0} \bar{\kappa} q^{\zeta-\frac{7}{4}}}\left[B_{x_{\mathrm{beg}}}\left(\zeta-\frac{7}{4}, \frac{1}{2}\right)-B_{x_{\mathrm{fin}}}\left(\zeta-\frac{7}{4}, \frac{1}{2}\right)\right]\right\}
$$

where $C=7.11 \times 10^{4} \mathrm{~km} \mathrm{~s}^{-1} \mathrm{AU}^{-\frac{11}{4}} \mathrm{day}^{2}, \mathcal{M}_{\text {beg }}=\mathcal{M}\left(t_{\text {beg }}\right)$, $\mathcal{M}_{\text {fin }}=\mathcal{M}\left(t_{\text {fin }}\right)$, and the incomplete beta function is

$$
B_{y}(\mu, \nu)=\int_{0}^{y} z^{\mu-1}(1-z)^{\nu-1} d z
$$

where $\mu>0, \nu>0$, and $0<y<1$. This definition requires a condition $\zeta>\frac{7}{4}$, which is always satisfied because $m>2$ and the second term on the right-hand side of Eq. (34) is positive.

We evaluated the exponential in Eq. (39) for four of the test comets in Table 7 (C/2001 Y4, C/2009 L5, $\mathrm{C} / 2006$ J9, and $\mathrm{C} / 2008 \mathrm{~K} 8$ ). When measured by $\mathcal{A}_{*}$, a mass loss over a $\sim 4$-hour long period, typically involved, was found from the ratios $\mathcal{M}_{\text {fin }} / \mathcal{M}_{\text {beg }}$ to be equivalent to a decrease in the effective dimensions by two to five orders of magnitude. However, this result overestimates the rate of mass drop because $\mathcal{A}_{*}$ is, as seen from Table 7 , dominated by the radial component $\mathcal{A}_{1}$. Since much of the mass of the nucleus of a dwarf sungrazer in this late stage of disintegration is reduced to expanding clouds of dust, including microscopic particles, a fraction of the detected acceleration is necessarily contributed by the Sun's radiation pressure. Loss effects due to erosion are more realistically estimated from the transverse and normal components, which include no contributions from solar radiation pressure. In that case the decrease in the effective nuclear size over the 4 hours is found to amount to $\frac{1}{2}$ to $2 \frac{1}{2}$ orders of magnitude, a result that suggests the objects' imminent decay. The heliocentric distances at which the test dwarf sungrazers were observed ranged from 14.5 to $8 R_{\odot}$.

The presence of nongravitational laws with slopes much steeper than the square of heliocentric distance even this close to the Sun (which is the case primarily with $\mathrm{C} / 2007 \mathrm{X} 3, \mathrm{C} / 2008 \mathrm{M} 4$, and C/2006 J9) does not necessarily rule out effects of radiation pressure, because the dust grains continue to fragment rapidly and the radiation-pressure acceleration varies inversely as the grain size, except for particles not exceeding in size a small fraction of a micron.

We are aware of the limitations that uncertainties in the astrometric data and short orbital arcs covered by the observations place on the quality of the orbit determina- tion. As a result, the orbits in Table 7 should be perceived with great caution. Observational errors are likely to be responsible for the two dynamically meaningless cases of negative $\mathcal{A}_{1}(\mathrm{C} / 2007 \mathrm{X} 3$ and $\mathrm{C} / 2008 \mathrm{M} 4)$. We also readily admit that the orbital sets and nongravitational parameters of all test sungrazers in Table 7 do not necessarily present unique solutions. On the other hand, we notice a high concentration of perihelion distances between 1.0 and $1.1 R_{\odot}$ among the nongravitational solutions in both Table 4 and 7 , which is significant. In the other elements, the deviations between the two sets of nongravitational solutions do not exceed a few degrees in $\omega$ and $\Omega$, but only $1^{\circ}$ in $i$, and 0.03 day in $t_{\pi}$.

\section{SUMMARY AND CONCLUSIONS}

The prime objective of this paper was to understand the discrepancy in the spatial orientation of apsidal lines between the bright and the dwarf members of the Kreutz sungrazing system, as revealed by their catalogued purely gravitational orbits. The apsidal lines of seven bright Kreutz sungrazers, observed from the ground in the years 1843-2011, are nearly perfectly aligned (to within a small fraction of $1^{\circ}$ ), whereas the apsidal lines of about 1600 faint, dwarf Kreutz sungrazers, detected only with the coronagraphs on board the SOHO and STEREO spacecraft between early 1996 and mid-2010, are distributed along an arc extending $\sim 25^{\circ}$ in perihelion latitude $B_{\pi}$ but not in perihelion longitude $L_{\pi}$, which is statistically invariable and equal to $L_{\pi}$ of the bright sungrazers. A corollary of this peculiar effect in a plot of the orbit inclination against the longitude of the ascending node is a distribution of the dwarf sungrazers along three parallel curves, each of which subtends an angle of about $15^{\circ}$ with the curve of the reference apsidal line, populated by the bright sungrazers, and passes through, respectively, the locations in the plot of comets C/1843 D1, C/1970 K1, and $\mathrm{C} / 2011 \mathrm{~W} 3$.

The differences between the apsidal-line orientation patterns of the dwarf and the bright Kreutz sungrazers suggest that the dwarf objects failed to avoid exposure to nongravitational dynamical forces that the bright ones managed to escape. From the perturbation theory we find that the dwarf sungrazers' extension of the apsidalline distribution in $B_{\pi}$ is a product of nongravitational accelerations, directed normal to the orbital plane. The broader is the range of these accelerations acting on individual dwarf sungrazers, the wider is the spread in the objects' angular elements.

We examined several different processes that could potentially generate the major effects in the latitude $B_{\pi}$. The only plausible trigger appears to be the erosiondriven transfer of momentum from outgassing to progressively fragmenting debris of the original nucleus of the dwarf sungrazer. This conceptual model deems fitting the motions of dwarf sungrazers by any purely gravitational orbit inappropriate and emphasizes the need to apply, instead, a nongravitational orbit whose apsidal line matches the reference apsidal line as closely as possible. For each dwarf sungrazer this condition requires (i) the incorporation of a nongravitational term with an unknown parameter into the equations of motion; (ii) the iteration of the orbital solution with the nongravitational term until a minimum offset from the reference apsidal line is reached; and (iii) the determination of a final set of 
orbital elements and the nongravitational parameter as products of the corrected apsidal-line offset. A successful implementation of this corrective procedure requires that the quality of fitting the astrometric observations (that is, their RMS residual) by the nongravitational solution be better than, or comparable to, that by the gravitational solution.

To examine the nature and the magnitudes of the nongravitational forces, we employed a sample of eight test dwarf sungrazers whose gravitational orbits were distributed nearly uniformly along a $\sim 90^{\circ}$-long arc in the longitude of the ascending node, between $305^{\circ}$ and $33^{\circ}$. The gravitational solutions left offsets from the reference apsidal line of up to $\sim 17^{\circ}$ (Table 4 ); to rectify these unacceptably large deviations, we proceeded in three steps.

In the first step, we applied the standard Style II formalism by Marsden et al. (1973) that incorporates the nongravitational terms into the equations of motion. Because of the strong trends in $B_{\pi}$, we focused on the normal component of the erosion-driven acceleration, which we introduced into the computations iteratively and minimized the offset of each dwarf sungrazer's orbit from the reference apsidal-line orientation to find the parameter $A_{3}$. We assumed in this step that the radial and transverse components of the nongravitational acceleration were nil, $A_{1}=A_{2}=0$. The resulting parameters $A_{3}$ for the eight test dwarf sungrazers were on the orders of $10^{-4}$ to $10^{-6} \mathrm{AU}$ day $^{-2}$, at least one order and up to three orders of magnitude greater than the largest values of $A_{1}$ among the non-Kreutz comets catalogued by Marsden \& Williams (2008). C/2007 X13 was found to be subjected to a nongravitational acceleration greater than the Sun's gravitational acceleration at the same heliocentric distance. The derived parameters approximately correlated with the offsets from the reference apsidal line left by the gravitational orbital solutions. These offsets were reduced by the nongravitational solutions substantially, never to exceed $1^{\circ} .4$.

Because Marsden et al.'s (1973) formalism employs a momentum-transfer law based on the assumption that the nongravitational acceleration is driven by outgassing of water ice, we compared, in our second step, this standard law with a few novel laws that describe outgassing of other species, more likely than water ice to match the sublimation conditions at extremely small distances, about $10 R_{\odot}$, from the Sun, where the dwarf Kreutz sungrazers are typically observed (footnote c to Table 7). The most powerful among the tested scenarios was the modified law, with a variable scaling distance $r_{0}$, linked to the degree of volatility of outgassing species. This exercise illustrates a great variety of behavior among the test sungrazers, from cases suggesting that outgassing is dominated by substances more refractory than forsterite to those with substances more volatile than sodium and almost as volatile as water ice. The magnitudes of the erosion-driven acceleration did not change substantially from those found in the first step. The test objects' apsidal-line offsets offered by the best fitting nongravitational laws were now further reduced to less than $0^{\circ} .7$.

The default condition, $A_{1}=A_{2}=0$, was relaxed in the third step, in which only the statistical constraint $\Delta L_{\pi}=0$ was still retained. The expansion from singlecomponent to three-component nongravitational solutions was computationally much more demanding, even though an employed relationship between the radial and transverse components meant an increase by only one parameter. For each of the test comets we used a solution based on the law that provided the least offset from the reference apsidal-line orientation, usually the modified law. The offsets for all eight tested sungrazers were now reduced to less than $0^{\circ} .2$, the level of intrinsic scatter in the orientation of the reference line of apsides among the bright sungrazers (Table 1); and, remarkably, in four cases the offset did not even exceed $0^{\circ} .01$.

Even though the RMS residual was not a criterion by which we judged the quality of orbital solutions, the results in Table 7 show that the final nongravitational sets of orbital elements provide a significantly better fit than the gravitational solutions (Table 4) in one case and slightly to moderately better fits in three cases, and that the fits are comparable in the remaining four cases.

The parameters $\mathcal{A}_{1}$ to $\mathcal{A}_{3}$ in Table 7 show that at a distance of $10 R_{\odot}$ from the Sun, all test dwarf sungrazers were subjected to nongravitational accelerations of more than 15 percent of the Sun's gravitational acceleration, or more than two orders of magnitude higher than implied by the peak outgassing-driven accelerations for the catalogued comets in nearly parabolic orbits.

There are two more points to emphasize. The first is that the eight test sungrazers do by no means represent a random sample. The condition that the orbits of the test objects be approximately uniformly distributed in the longitude of the ascending node required a careful selection of appropriate candidates. It was the small number of astrometric observations made with the $\mathrm{C} 2$ coronagraph that caused difficulties.

The second point has to do with the catalogued gravitational orbits of the dwarf Kreutz sungrazers. We required in this paper that for each selected test object the set of orbital elements computed by Marsden be closely reproduced by our code. This turned out to be more constraining than first thought, because in his effort to obtain a sungrazing-like orbit Marsden sometimes manipulated the values of the elements by assuming a particular value for the perihelion distance. The first author has been aware of Marsden's frustration with this issue for a number of $S O H O / S T E R E O$ sungrazers. He altogether too often obtained a perihelion distance smaller than the Sun's radius, which - especially in early years ${ }^{6}$ - he considered unphysical. On other occasions, the perihelion distance came out to be much too large, so that, without manipulation, he could not classify the object as a sungrazer in spite of the signature in the angular elements. Since Marsden's forcing a perihelion distance affected the other elements as well, all such cases curtailed the list of candidates for our test objects.

In this context, the strong concentration of perihelion distances just beyond $1 R_{\odot}$, apparent from Table 7 (as well as from the nongravitational solutions in Table 4) and already commented on, is notable. Given a scatter of $0.8 R_{\odot}$ in the perihelion distances from the gravitational orbits of the test sungrazers (Table 4), the apsidalline constraint should in no way cause the sharp peak in

6 Only after Sekanina's (2002) paper on the dynamical effects of cascading fragmentation among dwarf Kreutz sungrazers along their entire orbit about the Sun started Marsden gradually accepting the fact that the perihelion distances of these objects could be less than $1 R_{\odot}$. 
the distribution. It therefore appears that the introduction of the nongravitational terms into the equations of motion improved substantially the quality of orbit determination, even though the realistic errors in the elements in Table 7 are greater than the last decimal places carried. From comparison of the nongravitational sets of elements in Table 4 with those in Table 7 , we estimate that the errors are on the order of 0.01 day in $t_{\pi}$, from $0^{\circ} .1$ to a degree or so in $\omega$ and $\Omega$, from better than $0^{\circ} .1$ to nearly $1^{\circ}$ in $i$, and from better than $0.01 R_{\odot}$ to $\sim 0.1 R_{\odot}$ in $q$; the least well determined orbit appears to be that of C/2007 X3, which happens to be derived from only 7 astrometric observations.

Application of a basic mass-loss model for the test dwarf sungrazers suggests that while observed in the field of the SOHO's C2 coronagraph, along the terminal segment of their orbits, the nuclei fragmented copiously and their dimensions shrank at a dramatic rate, resulting in the objects' imminent decay, as illustrated by Schrijver et al. (2012) in the case of C/2011 N3. Along the radial direction, the nongravitational effect due to erosion-driven momentum transfer should, in the late stage of disintegration, have been enhanced, to at least a limited degree, by solar radiation pressure acting on the microscopic debris of the nucleus.

\section{WHAT TO DO NEXT?}

The results of our orbital analysis strongly suggest that due to a major erosion of their mass, the nuclei of dwarf Kreutz-system comets are in close proximity to the Sun subjected to a momentum-transfer effect of such magnitude that: (i) their motions cannot be fitted by employing the gravitational law alone, as such orbital solutions lead to grossly misleading results, and (ii) the apsidal-line orientations derived from such solutions require major corrective measures, whose application is involved and time consuming. Yet, the gravitational orbits are the only orbital data currently available for the dwarf Kreutz sungrazers. A perplexing issue is what to do to rectify this situation?

A skeptic would suggest that the quality of astrometric observations of the dwarf comets from SOHO/STEREO images is too poor to mount a massive project in an effort to fix the problem. In fact, reference to the low accuracy of the positional data has often been an argument used to question the merit of these objects' published orbital elements in the first place. Our results - although based on a very limited data sample - suggest that these doubts are not necessarily justified and that the problem can in principle be cured by appropriately accounting for the large erosion-driven nongravitational forces.

In practical terms, should the orbits of all, or in the least a sizable fraction, of the dwarf sungrazers of the Kreutz system be reanalyzed from scratch? The authors admit that they have neither resources nor a motiva- tion for getting involved with such a large-scale but essentially routine project that should basically follow the algorithm prescribed in this paper. Accordingly, we limit our comments to acknowledging that the current state of orbital analysis of the body of these objects is rather depressing, but we refrain from proposing a workable plan of action beyond merely recognizing that the task requires highly sophisticated computer-driven automation.

The authors thank A. Vitagliano for modifications in his code $E X O R B 7$ that he made at our request and thus allowed us to pursue all planned steps in our investigation. This research was carried out in part at the Jet Propulsion Laboratory, California Institute of Technology, under contract with the National Aeronautics and Space Administration.

\section{REFERENCES}

Biesecker, D. A., Lamy, P., St. Cyr, O. C., et al. 2002, Icarus, 157, 323

Brueckner, G. E., Howard, R. A., Koomen, M. J., et al. 1995, Sol. Phys., 162, 357

Ciaravella, A., Raymond, J. C., \& Giordano, S. 2010, ApJ, 713, 69 Danby, J. M. A. 1988, Fundamentals of Celestial Mechanics, p. 323 (2nd ed.; Richmond, VA: Willmann-Bell Publ., 466pp)

Gray, W. J. 2013, MPC, 84616 ff.

Hashimoto, A. 1990, Nature, 347, 53

Hicks, W. T. 1963, J. Chem. Phys., 38, 1873

Howard, R. A., Moses, J. D., Vourlidas, A., et al. 2008, Space Sci. Rev., 136, 67

Hufnagel, L. 1919, Astron. Nachr., 209, 17

Kimura, H., Mann, I., Biesecker, D. A., \& Jessberger, E. K. 2002, Icarus, 159, 529

Knight, M. M., A'Hearn, M. F., Biesecker, D. A., et al. 2010, AJ, 139,926

Kreutz, H. 1888, Publ. Sternw. Kiel, 3

Kreutz, H. 1891, Publ. Sternw. Kiel, 6

Kreutz, H. 1901, Astron. Abh., 1, 1

Marsden, B. G. 1967, AJ, 72, 1170

Marsden, B. G. 1970, IAU Circ., 2261

Marsden, B. G. 2005, ARA\&A, 43, 75

Marsden, B. G., \& Williams, G. V. 2008, Catalogue of Cometary

Orbits 2008, p. 108 (17th ed.; Cambridge, MA: Smithsonian Astrophysical Observatory, 195pp)

Marsden, B. G., Sekanina, Z., \& Yeomans, D. K. 1973, AJ, 78, 211

Nakano, S. 2001, Nakano Note 933

Nakano, S. 2006, Nakano Notes 1295 and 1305

Nakano, S. 2008, Nakano Note 1618

Schrijver, C. J., Brown, J. C., Battams, K., et al. 2012, Science, 335,324

Sekanina, Z. 1982, in Comets, ed. L. L. Wilkening (Tucson, AZ: University of Arizona), 251

Sekanina, Z. 2000, ApJ, 545, L69

Sekanina, Z. 2002, ApJ, 566, 577

Sekanina, Z. 2003, ApJ, 597, 1237

Sekanina, Z. 2005, Int. Comet Quart., 27, 225

Sekanina, Z., \& Chodas, P. W. 2007, ApJ, 663, 657

Sekanina, Z., \& Chodas, P. W. 2008, ApJ, 687, 1415

Sekanina, Z., \& Chodas, P. W. 2012, ApJ, 757, 127 (33pp)

Sekanina, Z., \& Kracht, R. 2013, ApJ, 778, 24 (13pp)

Sekanina, Z., \& Kracht, R. 2014, eprint arXiv:1404.5968

Thompson, W. T. 2009, Icarus, 200, 351 